\title{
Application of the Gaussian Stochastic Multiplicative Cascade Model to Choice Response Time Data
}

\author{
Rachel Heath \\ School of Psychological Sciences \\ University of Newcastle, Australia
}

\begin{abstract}
In two experiments involving a four-choice visual task, participants' response time difference series were shown to be significantly multifractal. This implied that information processing occurred on multiple time scales, and that performance was nonlinear rather than linear early in practice and whenever time constraints were placed on performance. This led to excellent fits to the multifractal spectra of a Gaussian multiplicative cascade model with two parameters, the mean and variance of the Gaussian stochastic process. As mean and variance were positively correlated, $Z m u$, the mean parameter estimated with reference to its estimate from 30 surrogate series, was used for data analysis. As practice progressed in Experiment 1, $\mathrm{Zmu}$, a measure of the multifractal component of the mean of the Gaussian process, decreased from the first to second practice session. Zmu increased when people were required to respond quickly in Experiment 2, suggesting its possible application as a measure of information processing capacity in a variety of neuropsychological and human factors applications. As a theoretical bonus, Zmu subsumes all of the sequential effects present in the response time series, thus obviating the need for the inclusion of a large number of additional parameters to represent these temporal dependencies.
\end{abstract}




\section{Introduction}

The choice response time (CRT) task is one of the oldest in psychology, forming part of the early studies on human decision making by Donders (translation from the Dutch, 1969). Despite over 150 years of research there has been no consensus on what model is best suited for accounting for, and predicting, the response time (RT) series observed in such tasks. Since the 1960s, researchers such as Bertelson (1961), Laming (1979), Rabbitt and Rodgers (1977) and Remington (1969) have investigated sequential effects in CRT tasks trying to understand how the RT on the present trial depends on stimulus-response contingencies occurring on at least the previous five trials (Luce, 1986). It is important to note that many of these tasks imposed a constraint on the time between response termination and presentation of the next stimulus, rather than using a fixed inter-stimulus interval (ISI) as was the case in the experiments reported in this paper.

Contemporary choice models, such as the drift diffusion model (DDM) (Ratcliff \& McKoon, 2008) and the Linear Ballistic Accumulator Model (LBAM) (Brown \& Heathcote, 2008), represent sequential RT effects by assuming that people adjust either the bias they have for one of several responses prior to the stimulus presentation, or by altering the rate of stimulus information acquisition for a stimulus that is thought more likely to be presented on a trial based on their experience with previous stimuli.

Even when model parameters mimic the sequential effects observed in RT data, there is the further task of determining the strategies people use to comply with the common conflicting requirement to respond as quickly and as accurately as possible. This is a difficult choice for participants when they expect their decisions to be more accurate the longer they take to process the stimulus information. In many cognitive tasks in which time to respond is not an issue, long RTs are often accompanied by a greater number of errors. As Vickers (1979) proposed, a dynamic adaptive adjustment to decision criteria might be the only way 
people can reasonably maintain compliance when overly rapid responses are penalised should they commit an error. This generally leads to an increase in RT on the next trial with the associated risk of the response exceeding subjective, or experimenter, imposed RT constraints.

To overcome some of the problems conventional choice RT models have in explaining sequential effects, Kelly, Heathcote, Heath and Longstaff (2001) designed an experiment in which people responded to the onset of one of four lights by pressing an associated button. In the first experiment, the inter-stimulus interval (ISI) was set equal to 1 sec for each of the 10 participants who took part in three experimental sessions. The error rate averaged across the ten subjects and the three sessions was low at $2 \%$. The RT data showed $\log -\log$ power-law spectra with a significant mean slope of -0.44 . This finding indicated that the RT series had a long memory, meaning that conventional ways to assess sequential effects, such as autocorrelation, may be inefficient when compared with more refined temporal analyses. For example, such data analysis techniques assume the presence of nonlinear dynamics in RT data, so that RT recorded at one point in time is not proportional to the previously recorded RTs, as would be the case if the RT time series were linear (Heath, 2000).

Nonlinear analyses using data from sessions two and three in the Kelly et al. (2001) study showed that noise-reduced RT series were low-dimensional with an estimated correlation dimension of about 2.8. This result meant that the RT dynamics leading to observed sequential effects could be represented within a three-dimensional space, but would not consume all of this space as would be expected if the underlying response process was completely random. Noise reduction is a useful technique to produce more accurate estimates of nonlinear dynamical indices. Evidence for nonlinearity in the RT series was obtained by showing that the prediction error was significantly less for the RT series when compared with 
surrogate series that shared the same linear properties as the RT series but none of the nonlinearity (Kelly et al., 2001, Figure 4). These surrogate methods are explained in more detail later in this paper. Overall, there was convincing evidence from $80 \%$ of the sessions that sequential RT data were the result of low-dimensional nonlinear, possibly chaotic, dynamics. In this new analysis of the RT data, we will examine whether these RT series might be multifractal, that is, fractal on all time scales. If this is the case, it would be interesting to see how well a multiplicative cascade model driven by a homogeneous Gaussian process can fit the estimated multifractal spectra. This type of model is explained in more detail in the Appendix and summarised below.

A second experiment using similar but more refined apparatus required 12 people to partake in three conditions, a self-paced (SP) CRT task with no response time constraints, a Fast condition in which the ISI was set at the participant's mean RT in the SP task, and a Slow condition in which the ISI was set at two standard deviations above the mean RT recorded in the SP task (Kelly et al., 2001). The average ISI was less in the Fast condition (449 $\mathrm{msec})$ than in the Slow condition $(578 \mathrm{msec})$, and more errors were made in the Fast condition $(5.2 \%)$ than in the Slow condition (3.1\%). However, these low error rates suggest that the task was still relatively easy.

The slope of the power spectrum when a logarithmic transformation was applied to the frequency and power scales, the log-log spectrum slope, was closer to zero for the SP condition $(-0.16)$, and significantly less in absolute value than the average slopes for the Fast and Slow conditions $(-0.28)$. So, time constraints that force people to respond more quickly reveal the presence of long-term temporal correlations in the RT data.

There was evidence of nonlinearity for all participants in the Fast condition, for $25 \%$ of the participants in the Slow condition, but for no one in the SP condition. So, a sufficiently demanding task is required to reveal nonlinearity in CRT data, one that forces people to 
respond more quickly than normal. It will be interesting to see what effect the reduction in information processing time has on the multiplicative cascade model parameters.

Although linear log-log spectra may arise from the superposition of linear processes, under certain conditions such spectra might provide evidence of fractal processes that are essentially the result of nonlinear processing (for a detailed account of the issues involved, see Wagenmakers, Farrell \& Ratcliff, 2004). There was evidence of such a fractal process in the Kelly et al. (2001) data as the correlation dimension was low and fractional in Experiment $1, \mathrm{M}=2.85, \mathrm{SD}=.53$, and in the Fast condition of Experiment $2, \mathrm{M}=2.56, \mathrm{SD}=.62$. Also, an estimate of the Hurst exponent, $H$, a measure of the long-range dependence between RT values over extended time periods, could be obtained from the absolute slope of the log-log spectrum, $s$, as $(1+s) / 2$. The estimates were 0.72 for Experiment 1 and 0.66 for the Fast condition of Experiment 2, suggesting that the RT series might be the result of fractional Brownian motion occurring in the underlying stimulus processing. As these values exceed 0.5, the Hurst exponent value for a diffusion process with independent increments, the associated diffusion process for these experimental conditions must contain positively correlated increments. Clearly, a more general nonlinear analysis of the RT dynamics is needed to clarify the origin of the observed sequential dependencies.

Wagenmakers, Farrell and Ratcliff (2004) proposed a fractionally integrated ARIMA model for RT series. This family of models assumes that each measure in an RT series can be represented by the weighted sum of previous values in the series together with their moving averages pooled over a fixed number of observations. These operations take account of sequential dependencies in RT data and smooth out any fluctuations. A fractional differencing parameter is used to allow persistence (large RTs following large RTs and viceversa) and anti-persistence (small RTs following large RTs and vice-versa), another type of sequential dependency observed in RT data. The fractional differencing parameter also 
allows for a trade-off between short-term persistence, as represented by ordinary autoregressive models, and long-term persistence that has often been suggested to be the basis for the log-log spectra observed in RT data (for a critique of this issue, see Wagenmakers, Farrell \& Ratcliff, 2005).

By replicating simple RT, choice RT and time estimation experiments, Wagenmakers, Farrell and Ratcliff (2004) showed that long-term persistence was more likely a cause of loglog spectra in tasks requiring strategic change, this occurring more often when one tries to maintain time estimation consistency over many trials. Of interest, Wagenmakers et al. (2004) also suggested that strategic changes in the response caution parameter of a Drift Diffusion Model (Ratcliff \& McKoon, 2008) may be sufficient to generate such RT series spectra.

On the other hand, Holden, Van Orden and Turvey (2009) used word pronunciation time data and their associated RT hazard functions, the probability of a response occurring in the next moment of time given that the response has not already occurred, to support a model based on the additive combination of power law and lognormal distributions, both of which are signatures of what they termed interaction dominant dynamics. These authors recognised the importance of constraints on cognitive performance that are responsible for generating the types of interactive multiplicity that might be revealed in speeded choice tasks. These ideas form part of the motivation for our developing and applying a model based on a hierarchical multiplicative process, a strong example of interaction dominant dynamics. As shown in the Appendix, a lognormal distribution plays an important role in hierarchical multiplicative models when each subprocess in the hierarchy is governed by a Gaussian process, a stochastic process similar to the one that drives the Drift Diffusion Model.

There is precedent for evaluating sequential cognitive behavior in terms of fractal nonlinear processes that can occur on multiple time scales. A familiar intuition obtains when 
a person has to contend with atmospheric turbulence while flying. You bounce up and down in your seat at a certain base frequency, your coffee flips back and forth at a higher frequency, the wings flap at a higher frequency still, but the activity with the highest frequency can be observed by watching the movement of control surfaces on the wing as they try to counteract the turbulence and maintain the aircraft in stable flight under autopilot control. It is worth noting that what you observe at these different simultaneous frequencies also occur on simultaneous time scales, ranging from the shortest temporal fluctuations on the wings to the longest as you bounce up and down on your seat. As activity at each of these time scales can be fractal, we have a complex system that is multifractal, an idea originally suggested by Mandelbrot in the 1960s (Mandelbrot, 1999).

As we will observe below, a multifractal process can be represented by its spectrum, which by analogy with the more familiar power spectrum, relates the magnitude of fractal processes according to their relative frequencies, just as we experience during aircraft turbulence. The multifractal spectrum is often an inverted $U$ shape characterised by its modal frequency and its width. For a homogeneous Gaussian multiplicative cascade, the mean is the location of the maximum of this spectrum, and the standard deviation is proportional to the width of the spectrum at some arbitrary location, such as zero. Being able to eyeball the Gaussian parameter estimates directly from the multifractal spectrum suggests we may be on the right modelling track!

The results reported in this paper are not the first time multifractal analysis has been used in cognitive psychology. Kutnetsov and Wallot (2011) showed evidence of multifractal scaling in 22 of 30 time-estimation sessions during which participants provided continuation estimates of time intervals under various feedback conditions following presentation of a series of exemplar time intervals of fixed duration. Although feedback following time interval estimation produced a reduction in the absolute value of the log-log spectrum slope, 
which approached zero suggestive of white noise, feedback had no effect on the width of the multifractal spectrum. This lack of a feedback effect occurred even when there was strong evidence for multifractality in the time estimation series. As feedback was provided in our experiments, these findings suggest that feedback may not affect the shape of multifractal spectra in choice RT tasks.

Ihlen and Vereijken (2010) considered cognition to involve an interactive system operating on various time scales, as opposed to a more conventional component-based system that compartmentalizes human-environmental interaction in terms of discrete linked processing stages. An example of the latter would be a cognitive system that considers veryshort term sensory memory, short-term memory and long-term memory as separate functional entities operating on different characteristic time scales and processing different kinds of information. This commonly used information processing representation can be contrasted with a more integrated system in which memory function is represented by intermittent processing among activities that occur on multiple time scales. So commonly used cognitive components, such as focus of attention, processing capacity and level of consciousness, may emerge quite naturally from a complex interactive information processing system.

A fundamental characteristic of many time series is intermittency, regular fluctuations interrupted by periods containing qualitatively different fluctuation rates. Such intermittency is illustrated by many real-world phenomena, such as neural firing bursts, stock market price fluctuations and earthquake rumblings (Ihlen \& Vereijken, 2010). In our aviation example, aircraft passengers often experience intermittency whenever there is atmospheric turbulence between periods of smooth flight. Intermittent RT series produced by fluctuations in response criteria, attention and many other cognitive variables might be expected to exhibit significant multifractality. 
In their multifractal analyses of RT data from Wagenmakers et al. (2004), Ihlen and Vereijken (2010) provided evidence for significant multiplicative interactions for the simple RT task. Unlike Kutnetsov and Wallot (2011), Ihlen and Vereijken found no evidence of such interactions for the time interval estimation task. Even in relatively simple cognitive tasks, periods of fast responding are interleaved with intermittent slow responding as attention fluctuates. Although reduced multifractality, as indicated by a narrowing of the multifractal spectrum, was more likely to be observed in a choice RT task than in a simple RT task, Ihlen and Vereijken (2010) showed that simple and choice RT data, as well as time estimation data from Wagenmakers et al. (2004), could be accounted for by a multiplicative cascade process exhibiting multifractal behavior.

In this paper we analyse performance in two experiments using a similar four-choice visual task, each session of which yields a series of responses and their RTs. Unlike conventional methods, the RT series are analysed without reference to which response has been made on each trial, so that correct and erroneous responses are analysed in the same series. In Experiment 1, the effect of practice is examined over three sessions for each of ten participants. When analysed in terms of the multifractal model we expect to find changes in the Gaussian mean and standard deviation parameters from the multiplicative cascade model that reflect improvement in performance resulting from experience at the task. In Experiment 2, using a different group of 12 participants, the effect of forcing participants to respond to a Fast or Slow stimulus presentation rate is expected to show how these Gaussian parameters are affected by cognitive workload. In both experiments, we expect the RT time series to be multifractal, especially when the cognitive processing demands placed on participants are high, such as early in practice and when the stimulus presentation rate is Fast.

First, we present a novel methodology for analyzing sequential choice RT data in terms of multifractal processes, including methods for estimating generalized Hurst functions 
and multifractal spectra. These methods are used to analyze data from the two experiments described in Kelly et al. (2001). The paper concludes with some speculative commentary on possible models for the observed multifractal outcomes and their practical implications for cognitive theory and applications.

\section{Applications of Multifractal Analysis in Choice Response Time Tasks}

The technology Kelly et al. (2001) used to detect nonlinearity in RT data involved a demonstration of monofractality. This occurred because only one attractor type on a single frequency time scale was revealed by nonlinear RT prediction. The latter uses a special type of shuffling of the RT series to remove its nonlinear content. This allows a prediction measure from the RT series to be compared with a distribution of predictions from these comparison series to determine whether the RT series is nonlinear. Prediction from the RT series was significantly better than chance for up to four future responses using surrogate control series with the same mean, variance and autocorrelation function as the RT series. As Ihlen and Vereijken (2013) showed in their analysis of choice RT data, the scale invariance suggested by linear log-log spectra, as was observed in the Kelly et al. (2001) data, might also be exhibited at many other time scales. When this occurs, there may be a complete spectrum of different scaling exponents that satisfy the requirements of a multifractal data representation. We will explore this possibility in the following analyses.

\section{A Novel Methodology for RT Time Series Analysis}

\section{Measuring the Fluctuations in RT Time Series}

All psychological series, including RT sequences, vary over time. Whereas autoregressive processes in which the current observation can be predicted by a linear combination of a finite series of previous observations are suitable for analysing short-range temporal processes, Detrended Fluctuation Analysis (DFA) is more useful to quantify the fractal nature of a time series that might be represented exclusively by long-range temporal processes. A 
fractal is a mathematical object that is scale-invariant (Mandelbrot, 1997). This means that a fractal object is similar in appearance irrespective of the time scale on which it is measured.

DFA compares each observation in a series, $x(i), i=1, N$, with the average of all observations, as indicated by the deviation score, $x(i)-\bar{x}$, the difference between the $\mathrm{i}$-th observation of a sequence of $N$ observations and their mean, $\bar{x}$. These deviation scores are added to yield a cumulative deviation score given by

$$
y(k)=\sum_{i=1}^{k}[x(i)-\bar{x}], k=1, N
$$

Interesting properties of the cumulative time sequence, $y(k)$, can be obtained by partitioning $y(k)$ into $N / n$ nonoverlapping subsequences of size $n$, fitting a linear segment to each subsequence to produce $y(n, k)$, and then computing the average fluctuation of the integrated series about the trend line (Peng, Havlin, Stanley \& Goldberger, 1995, Equation 1):

$$
F(n)=\sqrt{\frac{1}{N} \sum_{k=1}^{N}[y(k)-y(n, k)]^{2}}
$$

In many applications, $\log (F(n))$ is linearly related to $\log (n)$ with a slope, $\alpha$, that reflects the type of long-range dependency that exists in the original series. When $\alpha=0.5, x$ is an uncorrelated process. When $0.5<\alpha<1, x$ is a long-range persistent process in which large (small) values tend to be followed by large (small) values. When $0<\alpha<0.5, x$ is a longrange anti-persistent process in which large (small) values of $x$, tend to be followed by small (large) values. When the log-log spectrum slope equals $-1, \alpha=1$ (Peng et al., 1995). The slope parameter, $\alpha$, estimated using DFA is akin to the Hurst exponent, a measure of the longterm memory of a time series. An advantage of DFA and its extension described in this section to estimate multifractal spectra is that change in the process due to temporal 
fluctuations in the RT time series parameters can be accommodated by analysing the residuals following a polynomial regression fit to the trend line in Eq. (2).

As shown in the Appendix, a multifractal allows for a continuum of fractal processes, all intimately interconnected in a complex possibly hierarchical arrangement. Examples from the physical world include atmospheric turbulence and water flowing over a waterfall. Evidence for a multifractal process is obtained when the Hurst exponent is not a single value but depends on a multiplicity of interconnected fractal processes (see Kelty-Stephen, Palatinus, Saltzman \& Dixon, 2013, for a tutorial on multifractals).

Estimation of the multifractal properties of a time series can be achieved using a generalization of DFA. The scaling properties of each subsequence resulting from a partitioning of the original data series can be computed using a generalized version of DFA, MultiFractal Detrended Fluctuation Analysis (MFDFA) (Kandelhardt, Zschiegner, Koscielny-Bunde, Bunde, Havlin \& Stanley, 2002). The computational details for MFDFA are contained in the Appendix.

As a precursor to the stochastic multiplicative cascade model based on a diffusion-type Gaussian process that is described below, Ihlen (2013a) evaluated various ways to compute multifractal spectra using a simulated multifractal random walk as a benchmark (Bacry, Muzy \& Delour, 2001). The multifractal random walk is a stochastic process with stationary increments akin to fractional Brownian motion with Hurst exponent, $H$. The infinitesimal change in the stochastic activation function, $A(t)$, is represented by

$$
\delta B_{H}[A(t)]=B_{H}[A(t+1)]-B_{H}[A(t)],
$$

where $A(t)$ satisfies the requirements of a multifractal measure on the interval $[0, t]$ (Calvert \& Fisher, 2008, p.96 et seq.). With suitably chosen $A(t)$, the multifractal random walk exhibits intermittency accompanied by large and small fluctuations, just as people might experience 
when they are a passenger in an aircraft during turbulence that interrupts smooth flight. In a simulation driven by this stochastic model, Ihlen (2013b, p.923) showed that the width of the multifractal spectrum represents the relative extent to which the time series is dominated by periods of small and large fluctuations. As we will show, the maximum value of the multifractal spectrum occurs when the fluctuation frequency equals the expected value of $H$. When $A(t)$ results from a lognormal cascade across all time scales, the multifractal spectrum is symmetric, as shown in Figure 3 of Ihlen (2013b), and in the examples provided in the Appendix.

Ihlen's simulations showed that the most accurate reproduction of multifractal spectra produced by a lognormal cascade occurred when the multifractal spectrum was estimated using at least 4096 data points. However, the multifractal spectrum estimation methods evaluated by Ihlen (2013b) were sensitive to multiharmonic trends in the data, leading to significant prediction error. A recommended method to avoid the erroneous inference of multifractality is to compare the multifractal spectra and generalised Hurst functions estimated from the data with those estimated from surrogate series that contain no nonlinear interactions. The Appendix shows how surrogate methods, commonly used to detect nonlinearity in a time series (Heath, 2000), can be used for this purpose.

\section{METHOD}

In each of two experiments, the participant performed a four-choice task using visual stimuli. Short four-minute blocks of trials were used to minimize fatigue and to maximize the chance of consistent stationary performance within each block of trials. Except for the self-paced condition in Experiment 2, the trials within any block were presented at a fixed rate to maintain performance consistency and to allow for the appropriate application of nonlinear methodologies. 
The research protocol was approved by the Research Ethics Committee of the University of Newcastle, Australia. All subjects were volunteers who provided informed consent in accordance with international standards for research involving humans. Data from Experiments 1 and 2 were previously published as Kelly et al. (2001), to which the reader should refer for details of the selection of subjects, the experimental apparatus and the experimental design, as well as for an account of the basic results and analyses using novel nonlinear methods.

Data for this paper are contained in the Open Science Foundation Depository at https://osf.io/dvfmr/

\section{EXPERIMENT 1}

Ten people took part in three separate hour-long sessions involving a four-choice task in which stimuli were presented at a constant rate of one per second (interstimulus interval, ISI $=1 \mathrm{sec}$ ) regardless of when a response occurred. On each trial, the participant was required to indicate which light had been illuminated by depressing a spatially compatible response button. The stimulus appeared for $800 \mathrm{~ms}$ during which time the participant should have been able to make a response. After a delay of $200 \mathrm{~ms}$ the next stimulus appeared. Each session contained 11 blocks of 240 trials with a short rest period between blocks. RT was measured to $1 \mathrm{msec}$ accuracy. This was essentially a four-choice visual tracking task in which the participant's responses followed the changing stimulus location from one trial to the next.

\section{Results}

Using data from the final ten blocks of trials in each session, error percentage, averaged across the ten participants and the three sessions, was low at $2 \%$. The mean RT on error trials $(\mathrm{M}=323 \mathrm{msec}, \mathrm{SD}=50 \mathrm{msec})$ was less than that on correct trials $(\mathrm{M}=382 \mathrm{msec}, \mathrm{SD}=32$ $\mathrm{msec}$ ), the only significant difference over sessions being a decrease in mean RT for correct responses from session 1 to sessions 2 and 3 . 
Kelly et al. (2001) showed that the mean log-log spectrum slope averaged over each session and participant was -0.44 , there being no differences between sessions. Nonlinear analyses using data from sessions 2 and 3 showed that the noise-reduced RT series were lowdimensional with an estimated correlation dimension of about 2.8. This value implies that the RT dynamics can be represented within a three-dimensional space. Evidence for nonlinear dynamics in the RT series was obtained by showing that the nonlinear prediction error was significantly less for the RT series when compared with a similar error computed for the surrogate series that shared the same linear properties as the RT series but without the nonlinearity (Kelly et al, 2001, Figure 4). This result implies that there is more short-term nonlinear temporal structure in the original RT series compared to one that has had its nonlinear content removed.

Overall, there was convincing evidence that RT fluctuations are controlled by a lowdimensional nonlinear process. However, to achieve these results, the data had to be filtered to remove superimposed noise, leading to the possibility that the low-dimensional attractors were an artefact of this data manipulation. On the other hand, it was important to discover that the low-dimensional nonlinearity was observed when both correct and error trials were combined in the data analyses, as was also the case in the following multifractal analysis of these same RT series. It was interesting to note that when the error data were removed, the findings were only different for one session's data for one participant. So, analysing the intact RT series is an appropriate data analysis strategy when the emphasis is on response dynamics rather than response accuracy.

\section{Application of the Multiplicative Cascade Model to RT Series in Experiment 1}

The RT data for all ten participants were analyzed separately for each of the three sessions. To minimise the effect of autocorrelated information in each RT series, the differences between each successive RT were used as the input for a multifractal analysis using the 
Gaussian Multiplicative Cascade Model (GMCM), as described in detail in the Appendix. Figures 1(a)-(j) show the fit of the GMCM (solid black curve) to the multifractal spectra estimated from the data (black points). The corresponding mean values estimated from 30 surrogate series are shown by unfilled red dots, with the average multifractal spectrum for surrogates shown by the red line. Owing to the very close fits of the GMCM model, as indicated by the very small mean squared error (MSE) values, $95 \%$ confidence intervals for multifractal spectra points estimated from the surrogate series (shown as Control in the legend for each figure) are not shown in these figures. For some of the participants, the large differences between the multifractal spectra generated from the RT time series and those obtained from the surrogate series provided clear evidence that choice RT is governed by a stochastic multiplicative cascade process driven by a Gaussian. However, this difference tends to occur more often in the first practice session when the task is not so familiar and when both the RT mean and standard deviation are longer.

(a)
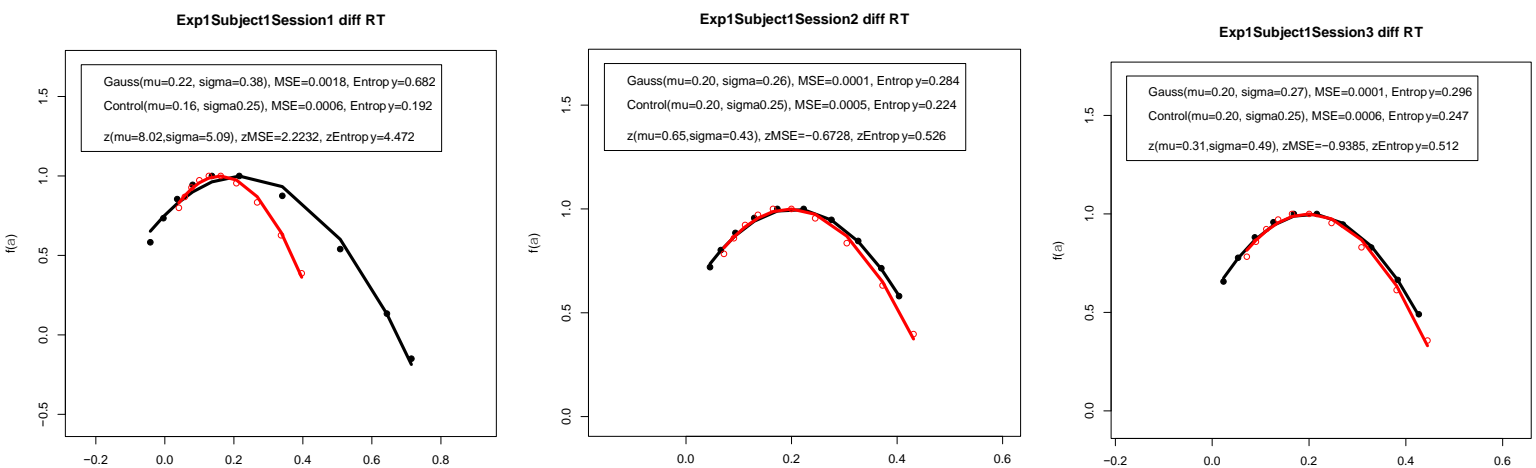


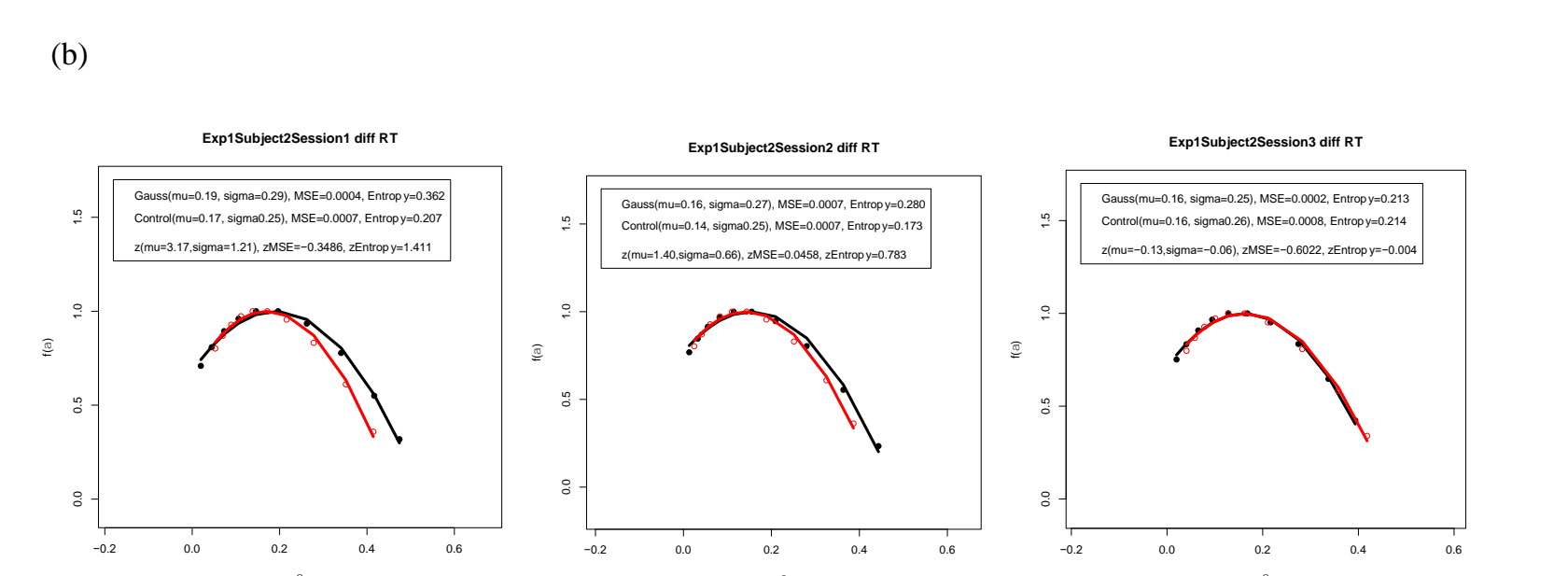

$$
\begin{aligned}
& \text { 言 } \\
& \text { 츠슨 }
\end{aligned}
$$




$$
\begin{aligned}
& \sqrt{\bar{\lambda}} \overline{\bar{\lambda}} \overline{\bar{n}} \\
& \text { 证证 } \\
& \text { 证言 }
\end{aligned}
$$


(h)
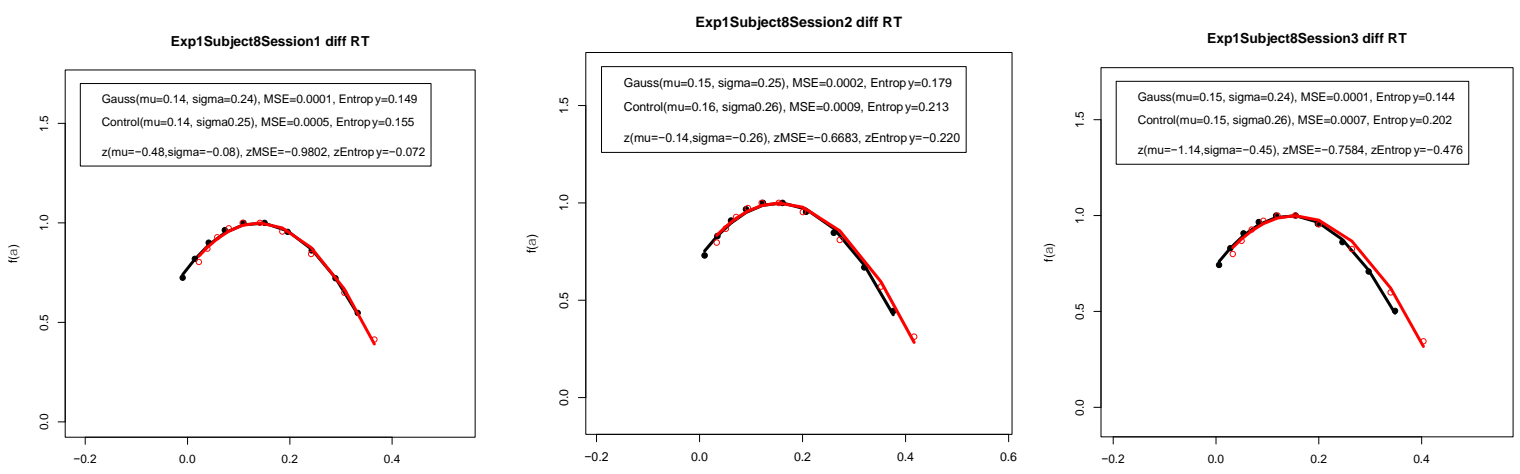

(i)
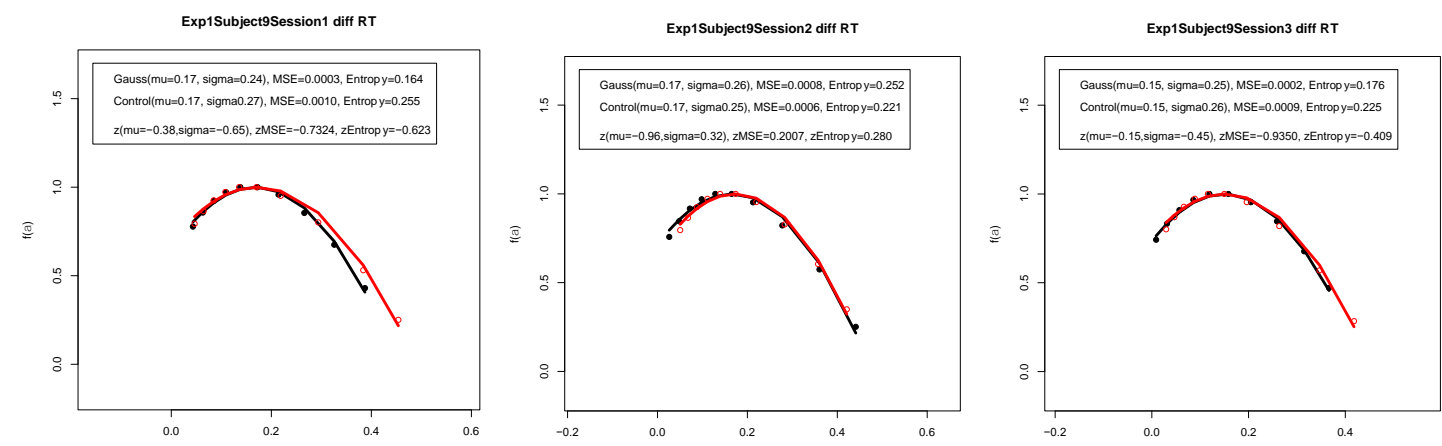

(j)
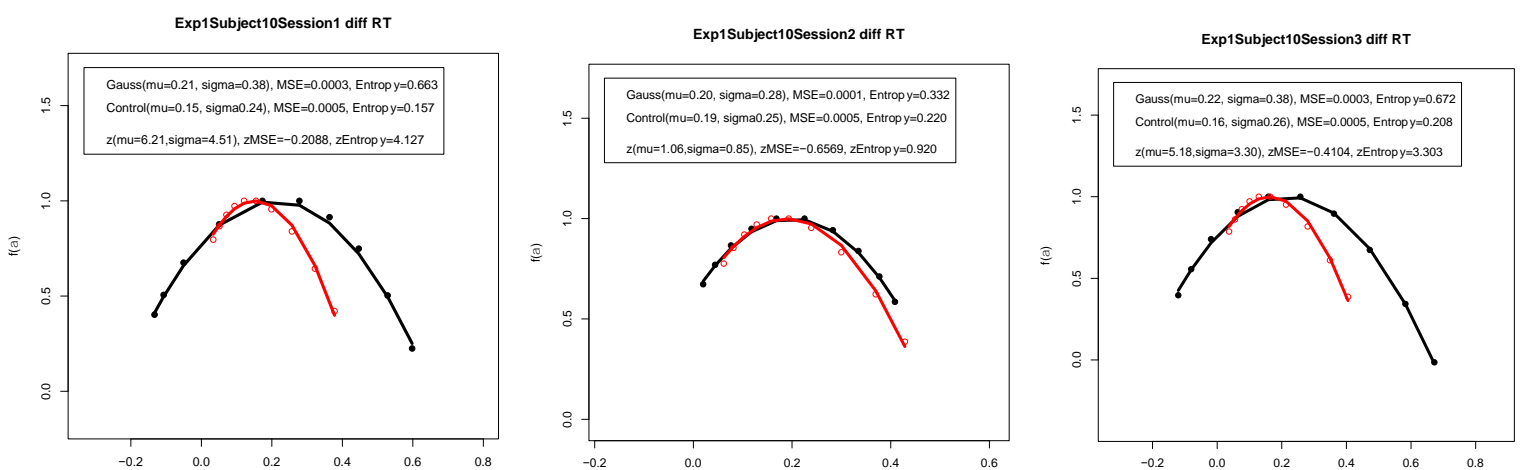

Figure 1. Multifractal spectra estimated from the differenced RT data for each of three sessions for Participants 1 to 10 in Experiment 1 . The data values are shown by the unfilled black circles and the corresponding surrogate values are shown by the red unfilled circles. The best-fitting multifractal spectrum predicted by the Gaussian Multiplicative Cascade Model is shown by the black curve for the pooled data and by the red curve for the average of the multifractal spectra produced by 30 surrogate control series. The legends for each graph show estimates of the model's parameters mu and sigma for the data and control series, as well as MSE goodness-offit values and estimates for the Entropy parameter. Values preceded by z in the legends are the standardized versions of these parameter estimates when the difference between the data estimate and the mean of the control series estimates is divided by the standard deviation of the control series parameter estimates. 
It is noteworthy that in every case, a Gaussian Multiplicative Cascade model fit the multifractal spectra very closely for the original RT series and for the average surrogate series. In the following analyses, the Type I error rate was set at 0.05 and the Holm correction was used for multiple post-hoc tests. Bayes Factor analyses supported the results obtained by conventional means and are not reported.

Mean RT decreased over sessions from $401 \mathrm{msec}$ in Session 1 to $380 \mathrm{msec}$ in Session 2 and $370 \mathrm{msec}$ in Session 3, $F(2,18)=11.09, p<.001$. There were significant differences between Session 1 and both Session $2(p=0.013)$ and Session $3(p<.001)$, but no difference between Session 2 and Session $3(p=0.138)$. The RT standard deviation, SD RT, decreased over the three sessions from $78 \mathrm{~ms}$ to $65 \mathrm{~ms}$ and $60 \mathrm{~ms}, F(2,18)=28.55, p<.001$. There were significant differences between Session 1 and both Session $2(p<.001)$ and Session $3(p$ $<.001)$, as well as between Session 2 and Session $3(p=0.04)$. These significant differences are shown in Figure 2.
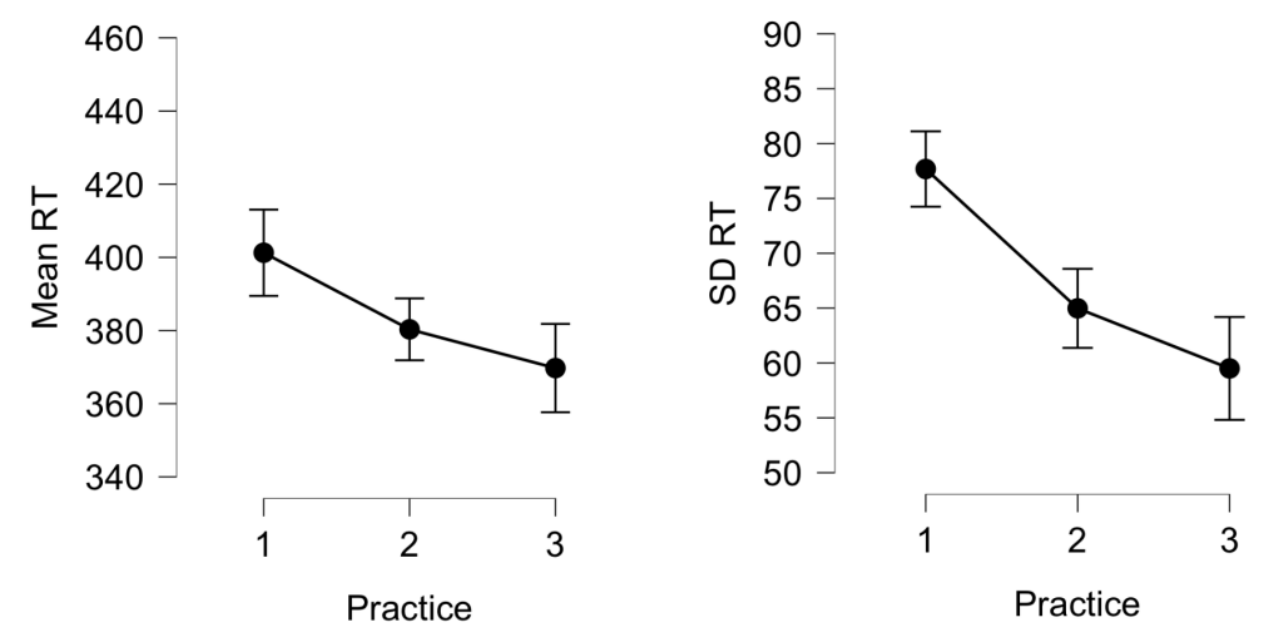

Figure 2. Mean RT (msec) and RT standard deviation (msec) over three practice sessions in Experiment 1.

The estimate of $m u$ changed over sessions from 0.171 to 0.156 and $0.160, F(2,18)=$ $4.08, p=0.034$. This was the result of a significant decrease in $m u$ from Session 1 to Session $2(p=0.039)$. No other between-session difference was significant. The estimate of sigma changed over sessions from 0.298 to 0.256 and $0.273, F(2,18)=4.66, p=0.023$. This was the 
result of a significant decrease in sigma from Session 1 to Session $2(p=0.022)$. No other between-session difference was significant. The correlations between the $m u$ and sigma estimates were $0.84, p=.003$, for Session $1,0.74, p=.015$, for Session 2 and $0.73, p=.017$, for Session 3. It is worth noting that the ratio of $m u$ to sigma, a measure of signal detectability akin to $d$ ' of Signal Detection Theory, did not change much with practice, the ratio of means being $0.57,0.61$ and 0.59 across the three sessions. The significant differences in $m u$ and sigma between Sessions 1 and 2 are shown in Figure 3.
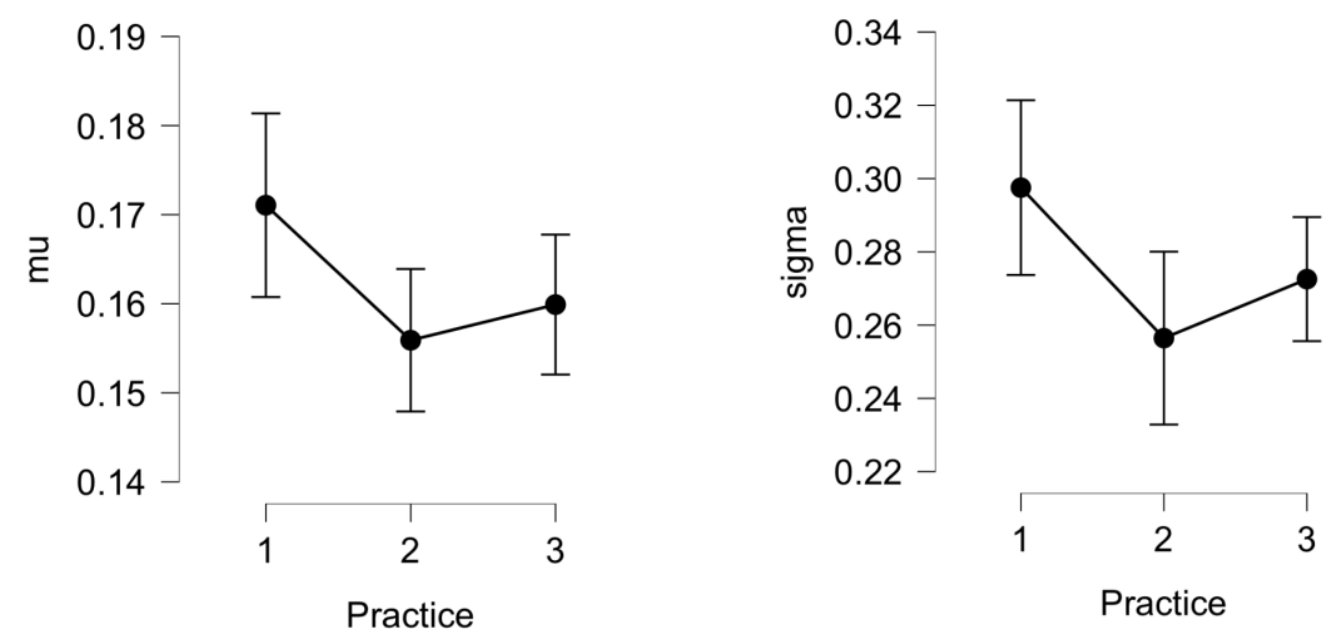

Figure 3. Mean values of the Gaussian Multiplicative Cascade parameters, $m u$ and sigma, for the three practce sessions in. Experiment 1 . The $95 \%$ confidence intervals are shown for each estimate.

As shown in the Appendix, the Entropy of a log-normal distribution provides a convenient index of information processing capacity for the GMCM. Entropy was estimated by substituting the estimates of $m u(\mu)$ and sigma $(\sigma)$ into

$$
E(\mu, \sigma)=\mu+\frac{\ln \left(2 \pi \sigma^{2}\right)+1}{2}
$$

A significant effect of practice at the task over three one-hour sessions was obtained for Entropy, $F(2,18)=5.36, p=0.015$, with post-hoc comparisons showing a significant decrease in Entropy between the first and second sessions, $t(9)=3.25, p=0.013$. This result 
is to be expected as Entropy decreases as mu and sigma decrease. This significant effect of Practice is shown in Figure 4.

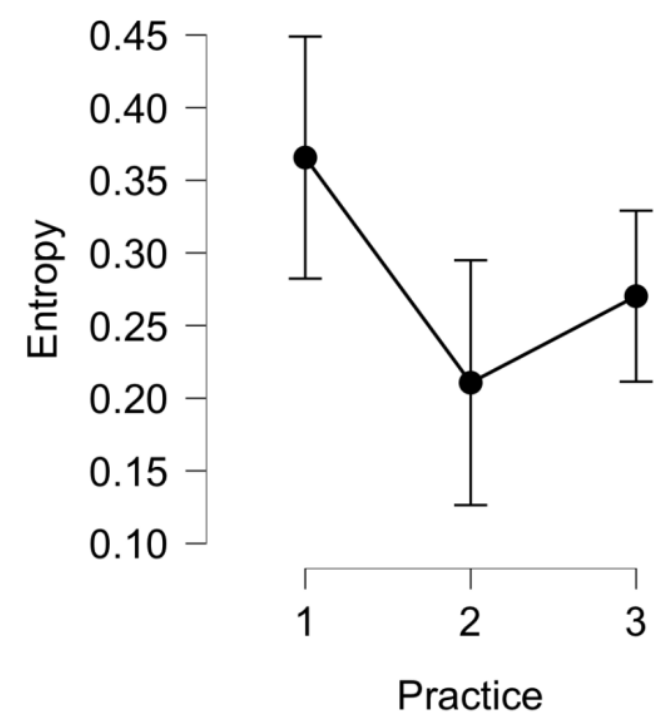

Figure 4. Mean values of Entropy estimated using the Gaussian Multiplicative Cascade parameters, $m u$ and sigma, for the three practce sessions in Experiment 1. The 95\% confidence intervals are shown for each estimate.

A standardised score, $Z m u$, was computed by taking the difference between $m u$ from the data and its mean value based on the surrogate samples, and dividing this difference by the standard deviation of the estimates of $m u$ obtained from the surrogate samples. When it is greater than or equal to $1.65, \mathrm{Zmu}$ lies outside the one-tailed $95 \%$ confidence interval for estimates obtained from the surrogate series, so the original RT series is likely to be multifractal. A one-tailed test is appropriate as Zmu can only be positive if the multifractal hypothesis is true. The mean values of $Z m u$ for the three sessions are 2.73, 0.29 and 0.99 , respectively, indicating that the RT series are likely to be multifractal only for the first Session, these differences being statistically significant, $F(2,18)=5.27, p=0.016$. Only the difference between Sessions 1 and 2 was significant following post-hoc tests $(p=0.017)$. When this analysis was repeated for Zsigma, the mean values were 1.64, 0.11, 0.74, with a significant difference between Sessions 1 and $2(p=0.016)$ following a significant analysis of 
variance using a within-subjects design, $F(2,18)=5.03, p=0.018$. These findings are shown in Figure 5.
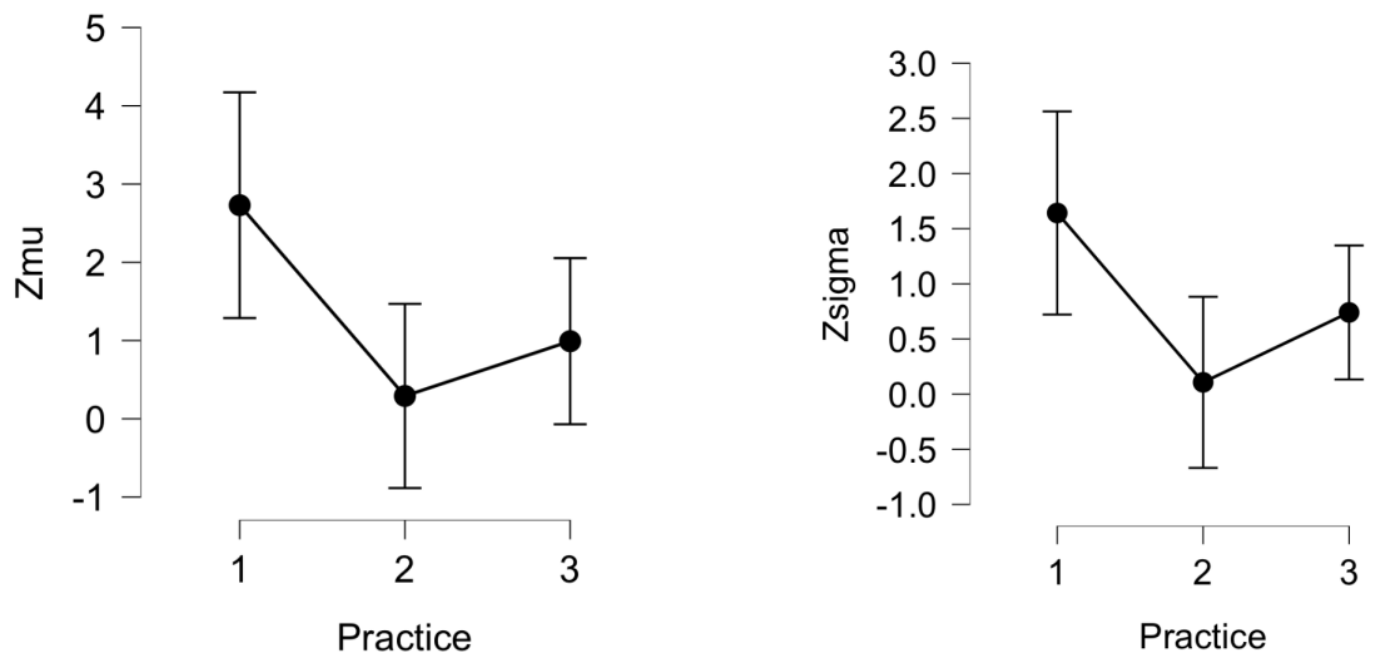

Figure 5. Mean values of the $\mathrm{Z}$ transforms of the Gaussian Multiplicative Cascade parameters, mu and sigma, Zmu and Zsigma, respectively, for the three practce sessions in. Experiment 1 . The $95 \%$ confidence intervals are shown for each estimate.

\section{Discussion}

The results from Experiment 1 provided a more complete picture of the complex dynamics in RT series than had been revealed by a previous analysis of these data by Kelly et al. (2001). Using the one-tail $95 \%$ confidence $z$ value of 1.65 for any of Zmu and Zsigma in the fit of the GMCM as suitable evidence of a multifractal process in a four-choice task, 7 out of 10 participants showed significant multifractal processes for session 1, 1 participant for Session 2 and 3 participants for Session 3. Practice at this relatively easy task for which the mean RT was well within the response deadline period of one second, resulted in a shorter mean RT, lower values of $m u$ and sigma, and the RT time series becoming monofractal rather than multifractal.

Clues to the nature of these multifractal processes that occurred in the early stages of practice at the task were provided by the prominent right-symmetric shape in many of the multifractal spectra shown in Figure 1, as well as the prominent difference between the data and surrogates in the right side of the multifractal spectrum. This finding suggests that the 
processes that generate the RT series operate at short time scales within a trial and possibly over several previous trials, as has been confirmed in numerous studies of sequential effects in RT data (Laming, 1979; Luce, 1986; Rabbitt \& Rogers, 1977; Remington, 1969).

All empirical multifractal spectra were fit well by the Gaussian model for all 10 participants. There was a statistically significant decrease in the Gaussian mean parameter and a corresponding decrease in the Gaussian standard deviation parameter following practice at the task. This finding is equivalent in theory at least to a decrease in the location of the maximum and width of the multifractal spectrum during extended practice. It is amazing that one can simply read off the mean value for the stochastic generator of a complicated multiplicative cascade process by inspection of the multifractal spectrum. Application of multifractal analyses to EEG data has shown that the peak location of the multifractal spectrum decreases as the level of consciousness increases from deep sleep to the awake state (Torick \& Mandelkern, 2013). According to the Gaussian multiplicative cascade model, this result implies that the mean of the Gaussian process driving the multiplicative cascade may be inversely related to a person's level of consciousness. So by analogy, an effect of practice at a task might be to make the task more autonomous so that it requires less conscious effort.

We now consider the effect of processing time on the fit of the GMCM as well on the parameters $m u$ and sigma by varying the time allowed for the participants to perform the four-choice task. As Experiment 1 showed that practice effects are essentially eliminated after a one-hour session, in Experiment 2 the first session was used to determine appropriate deadlines for the slow and fast sessions to follow. It was expected that estimates of the Gaussian parameters may provide insight into, as well as quantification of, information processing capacity in speeded choice tasks. 


\section{EXPERIMENT 2}

In Experiment 2, 12 participants took part in three experimental conditions using a fourchoice task similar to that used in Experiment 1, except that the stimuli were square patches located on a computer screen, rather than light onsets, as was the case in Experiment 1. In Experiment 2 the timing precision was increased to $0.1 \mathrm{msec}$. In the Self-Paced condition, each participant was required to respond as quickly and as accurately as possible in accordance with commonly used instructions in RT tasks. The other two conditions were devised individually for each participant based on the mean and standard deviation of their response times in the Self-Paced condition. In the Fast condition, stimuli were presented with a constant ISI equal to that participant's mean RT in the Self-Paced condition. In the Slow condition, stimuli were presented with a constant ISI equal to that participant's mean RT plus two standard deviations, as determined from performance in the Self-Paced condition. Each experimental session contained 13 blocks of 240 trials.

\section{Results}

Based on the defining characteristics of the two Paced conditions, Kelly et al., (2001) showed that the average ISI was less in the Fast condition $(449 \mathrm{msec})$ than in the Slow condition (578 msec). More errors were made in the Fast condition (5.2\%) than in the Slow condition (3.1\%). Mean RT was significantly less for errors than for correct responses in all three conditions. As expected, the Fast condition was more demanding than the Slow condition. It was worth noting that in the Fast condition, RT was consistently less following an error, $F(1,11)=56.07, p<.001, \mathrm{MSE}=79.04$.

The Mean RT and SD RT results for each of the three experimental conditions are shown in Figure 6. Using repeated measures ANOVAs, there was a significant decrease in Mean RT, $F(2,22)=47.82, p<.001$, with significant differences between each possible pair of the Self-Paced, Slow and Fast conditions as determined by the Holm post-hoc test. These 
are shown by the lack of overlap between the $95 \%$ confidence intervals in the left graph in Figure 6.

As shown in the right graph in Figure 6, there was a marginal decrease in the RT standard deviation (SD RT) between the three experimental conditions, $F(2,22)=3.61, p=$ .044 , but no significant differences between pairs of conditions using post-hoc tests. In particular, there was no difference between SD RT for the Slow and Fast conditions. So, the speeded responses in the Fast condition produced a lower mean RT than for the Slow condition but no difference in the RT standard deviation.
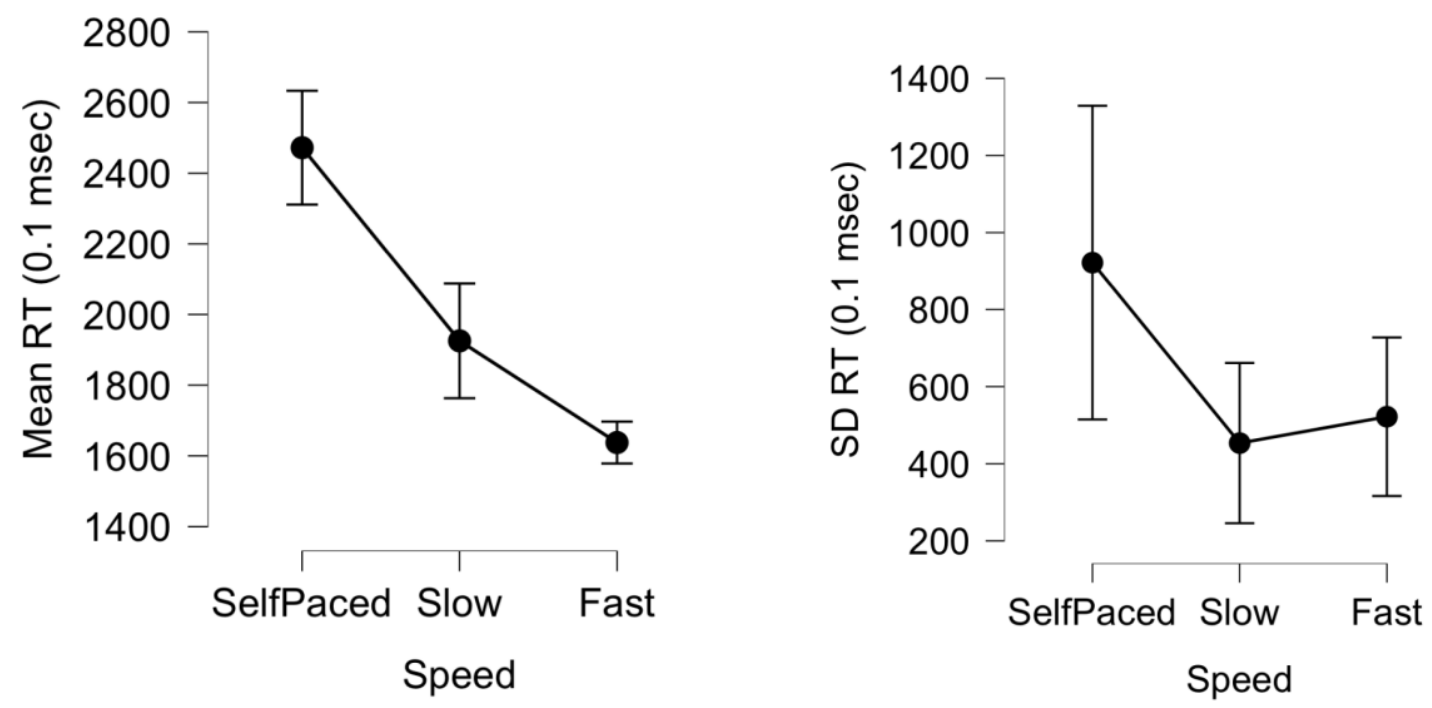

Figure 6. Mean and SD RT for the Self-Paced, Slow and Fast conditions in Experiment 2. RT is measured to 0.1 msec accuracy and $95 \%$ confidence intervals are shown for each point.

\section{Application of the Gaussian Multiplicative Cascade Model to the RT Data from}

\section{Experiment 2}

Prior to multifractal analysis, successive RT values were differenced for each of the three response speed conditions to minimise the effect of autocorrelated information in each RT series. As shown in Figure 7 (a) - (1), the fit of the Gaussian multiplicative cascade model was extremely close for all 36 multifractal spectra for the SelfPaced, Slow and Fast conditions. In almost all these cases, the difference between the multifractal spectrum 
estimated from the differenced RT data and the corresponding multifractal spectrum estimated from the 30 surrogate series when no nonlinearity was present, provided strong evidence for multifractality in this four-choice choice task.

(a)
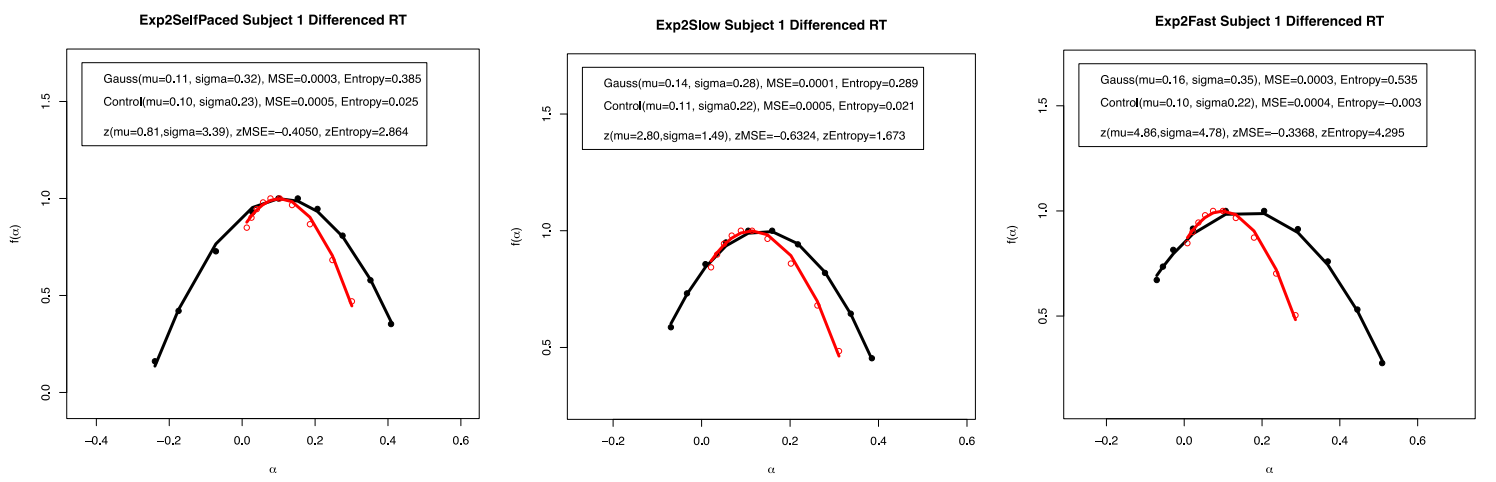

(b)
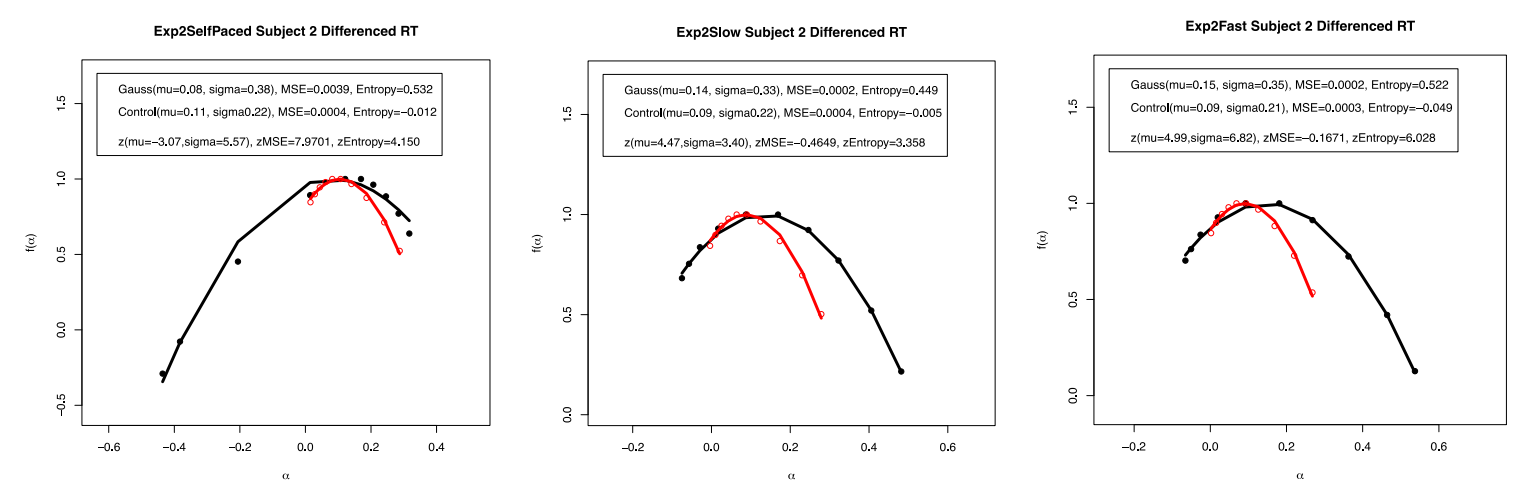

(c)
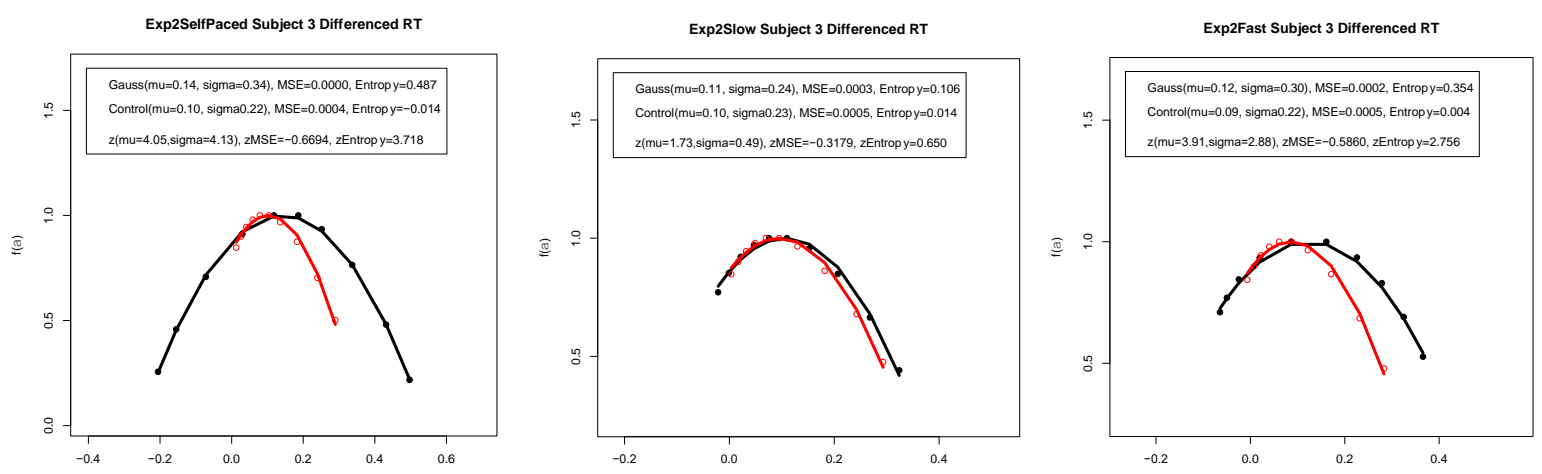


$$
\begin{aligned}
& \text { 辰层 } \\
& \text { 르슬 }
\end{aligned}
$$

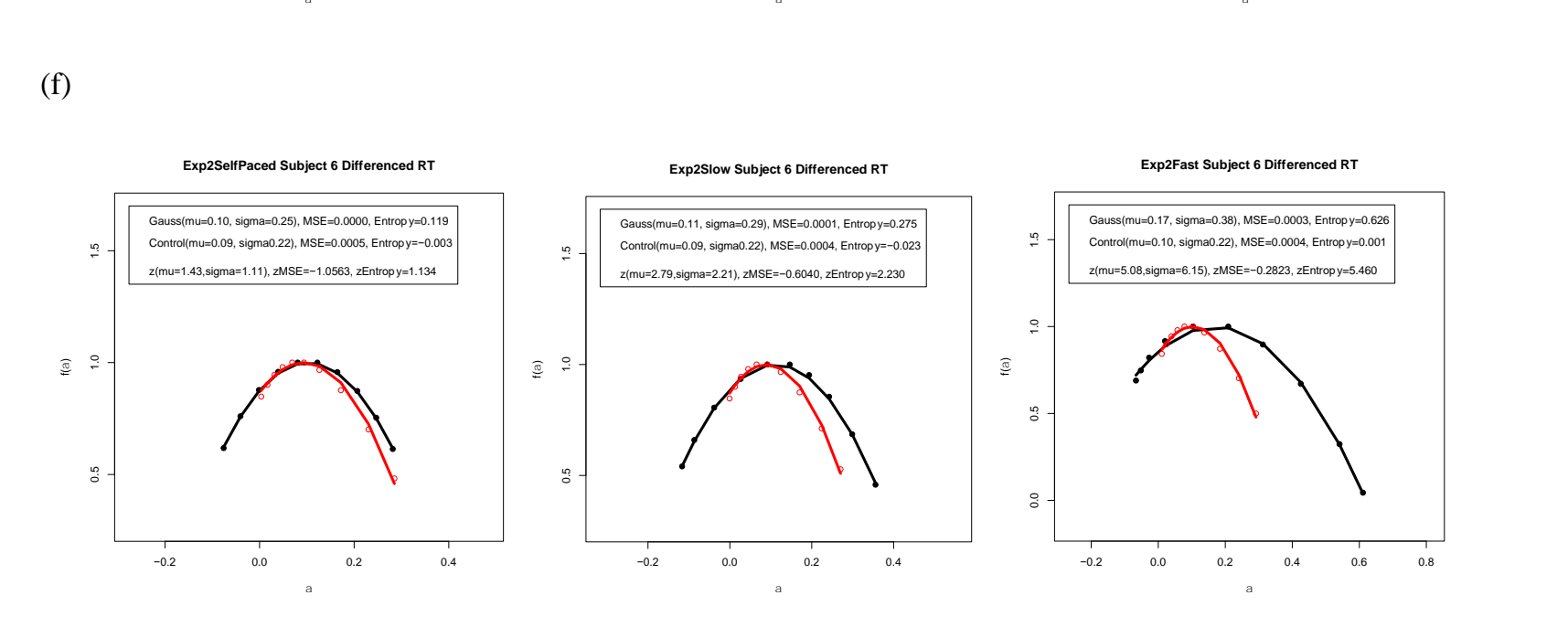




$$
\begin{aligned}
& \sqrt{\overline{2}} \\
& \sqrt{\overline{1}} \overline{\bar{a}} \\
& \text { 语 }
\end{aligned}
$$



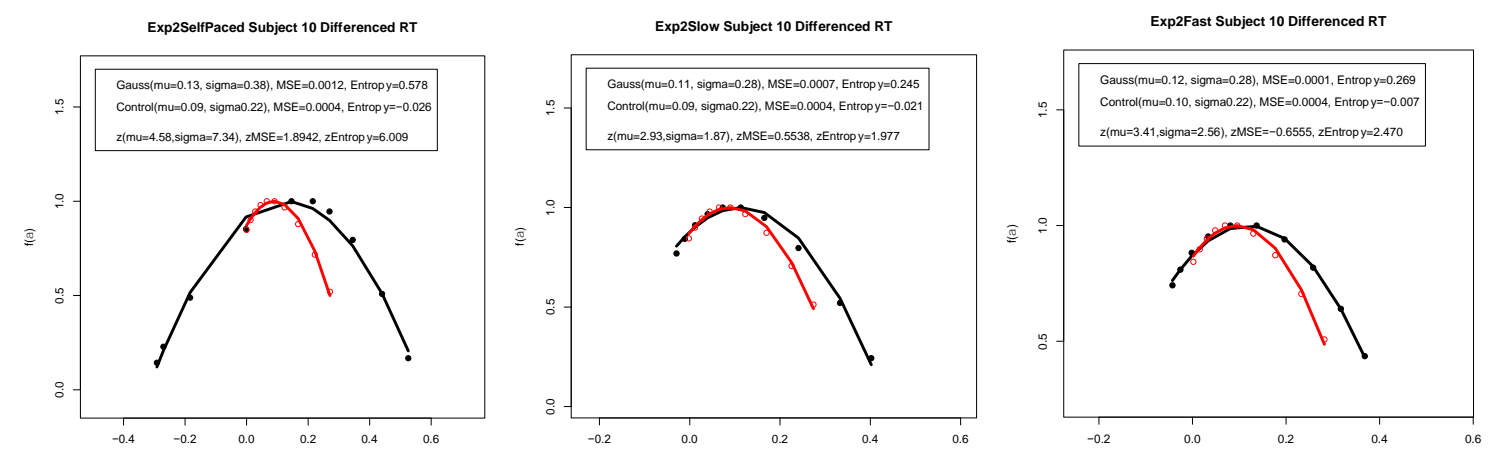

(k)
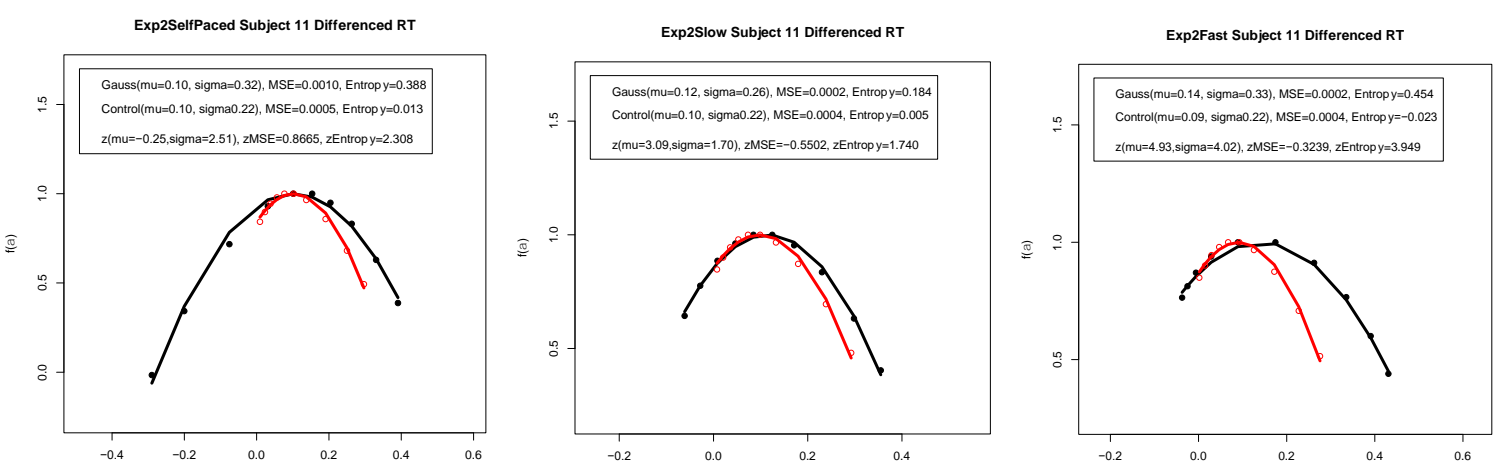

(1)
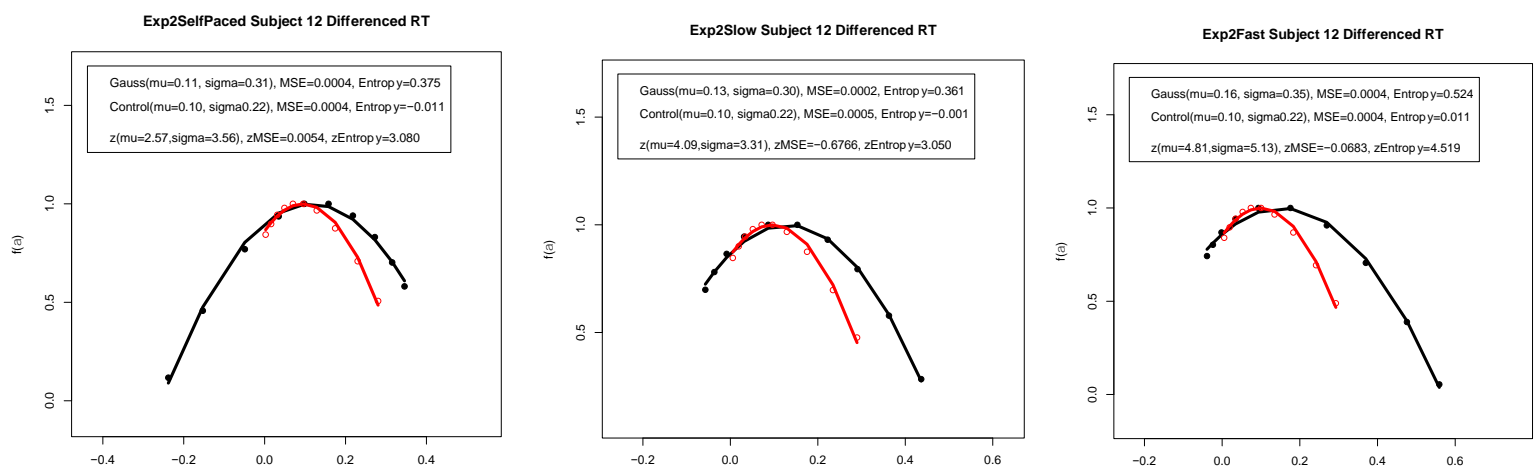

Figure 7. Fits of the Gaussian Multiplicative Cascade model (GMCM) to multifractal spectra estimated from RT difference data from the Self-Paced, Slow and Fast sessions for Participants 1 to 12 in Experiment 2. The data values are shown by the filled black circles and the corresponding surrogate values are shown by the red unfilled circles. The black curve shows the multifractal spectrum predicted by the best-fitting GMCM to the data and the red curve shows the multifractal spectrum predicted by the best-fitting GMCM to the average of the surrogate data. The legends for each graph show estimates of the model's parameters mu and sigma for the data and control series, as well as MSE goodness-of-fit values and estimates for the Entropy parameter. Values preceded by $z$ in the legends are the standardized versions of these parameter estimates when the difference between the data estinate and the mean of the control series estimates are divided by the standard deviation of the control series parameter estimates. 
In the following Analyses of Variance, data from all 12 participants across the three experimental conditions, Fast, Slow and Self-Paced (SP) were analyzed. To test the GMCM, paired-sample t-tests were performed for only the Fast and Slow conditions. Processing speed had a statistically significant efffect on $m u, t(11)=6.66, p<.001$, with a mean of 0.122 for the Slow condition and 0.15 for the Fast condition. Processing speed also had a statistically significant effect on sigma, $t(11)=7.92, p<.001$, with a mean of 0.283 for the Slow condition and 0.345 for the Fast condition. These findings, shown in Figure 8, indicate how increases in $m u$ and sigma result from a decrease in information processing time.
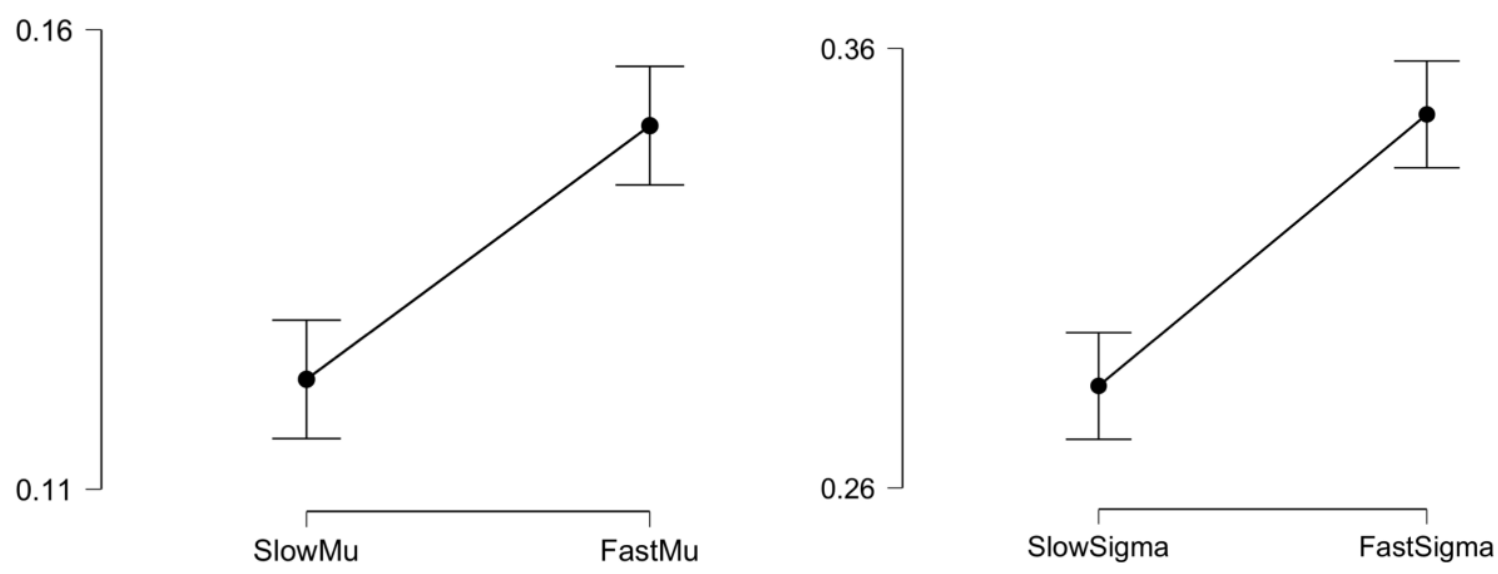

Figure 8. Changes in the Gaussian Multiplicative Cascade Model's $m и$ (left graph) and sigma (right graph) parameters with an decrease in response time speed in Experiment 2. 95\% confidence intervals are shown for each mean $m u$ and sigma estimate.

Although estimated as independent parameters when fitting the GMCM, the correlations between $m u$ and sigma were 0.85 for the Self-Paced task, 0.87 for the Slow task and 0.94 for the Fast task, all highly significant, $p<.001$. So the effect of information processing demand would be expected to be similar for both parameters, as shown in Figure 8.

A more sensitive measure of information processing demand is obtained by computing the difference between each GMCM parameter and its mean estimated from 30 
surrogate series and dividing this difference by the standard deviation of the GMCM parameter estimates obtained from the surrogate series. This produces $Z m u$ corresponding to mu, Zsigma corresponding to sigma and ZEntropy corresponding to Entropy. As shown in Eq. (A8) in the Appendix, Entropy is derived from the $m u$ and sigma parameters as the information content in a log-normal distribution that is obtained in the limit following information processing by a Gaussian Multiplicative Cascade.

Processing speed had a significant effect on $Z m u, t(11)=5.18, p<.001$, with a mean of 3.12 for the Slow condition and 4.62 for the Fast condition. Processing speed also had a significant effect on Zsigma, $t(11)=5.28, p<.001$, with a mean of 2.75 for the Slow condition and 5.43 for the Fast condition, and ZEntropy, $t(11)=3.34, p=0.007$, with a mean of 2.81 for the Slow condition and 4.86 for the Fast condition. These findings, shown in Figure 9, suggest a strong effect of the decrease in information processing time in the Fast condition.
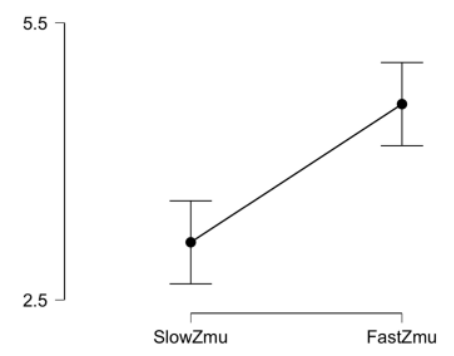
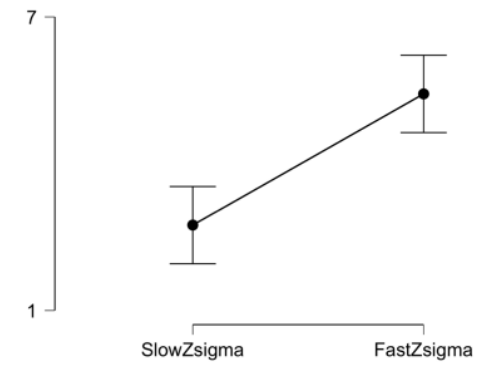
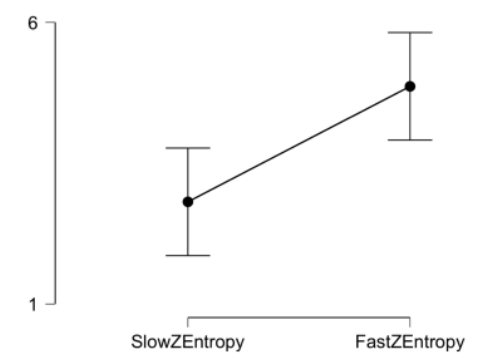

Figure 9. Changes in the Gaussian Multiplicative Cascade Model Z scores, Zmu (left graph), Zsigma (centre graph) and ZEntropy (right graph), with an increase in response time speed in Experiment 2. 95\% confidence intervals are shown for each parameter estimate.

A logistic transformation of the proportion of correct responses, $\operatorname{Logit}(P C)$ is defined by

$$
\operatorname{Logit}(P C)=\log \left(\frac{P C}{1-P C}\right)
$$

Figure 10 shows the relationship between Mean RT and $\operatorname{Logit}(P C)$ for the Fast (red points) and Slow (green points) deadline conditions in Experiment 2. The figure shows the bestfitting line when Mean RT is predicted from LogitPC with a slope of 23.14 and an intercept 
of 118.9. The fit is statistically significant, $F(1,22)=29.39, p<.001$, with adjusted $R^{2}=0.57$. This strong linear relationship between Mean $R T$ and $\operatorname{Logit}(P C)$ has also been shown to be a consequence of a generalised random model for decision-making (Link, 1975, Link \& Heath, 1975).

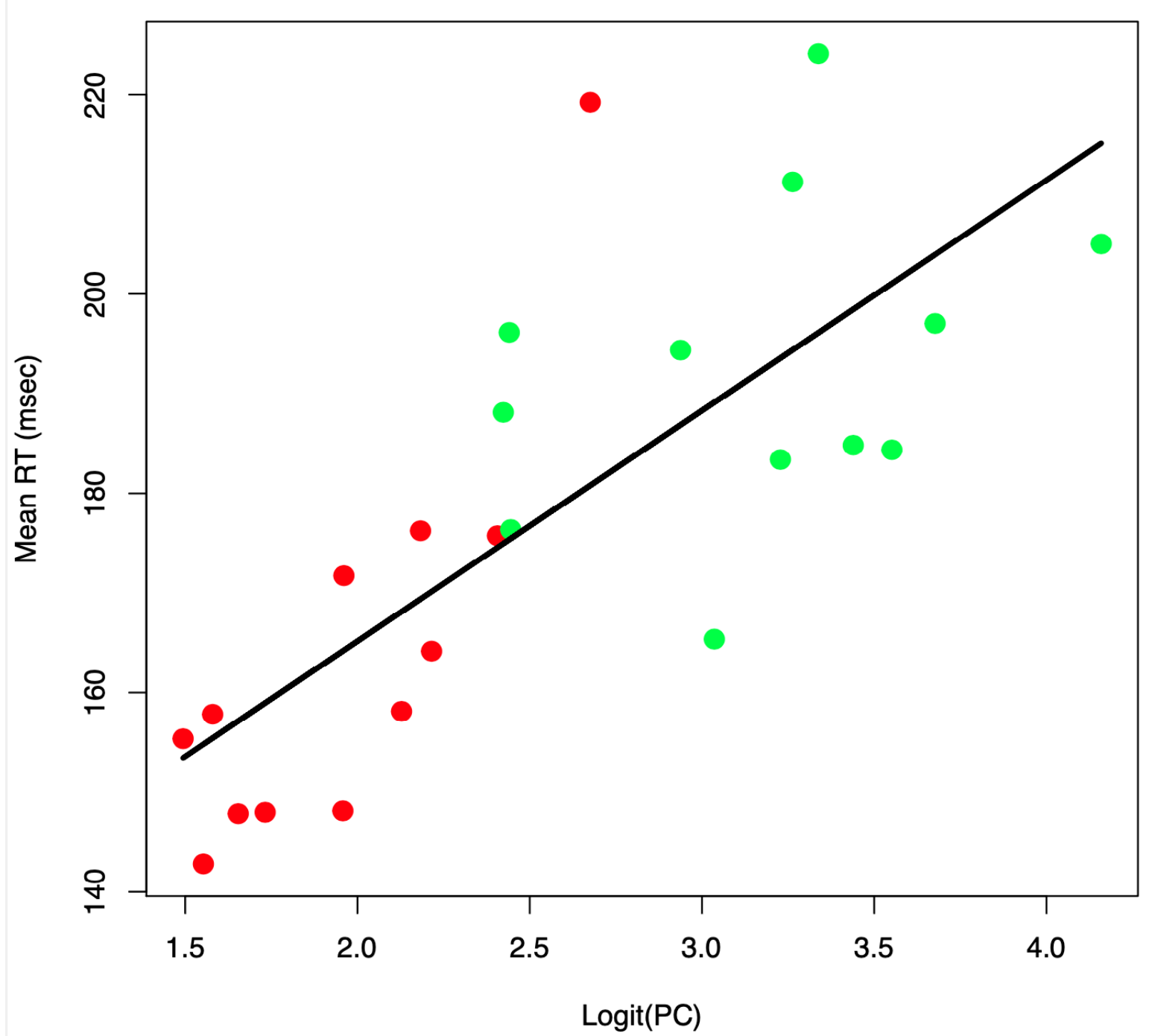

Figure 10. The relationship between mean RT and Logit(PC) for each participant in the Fast (red points) and Slow (green Points) conditions of Experiment 2. 

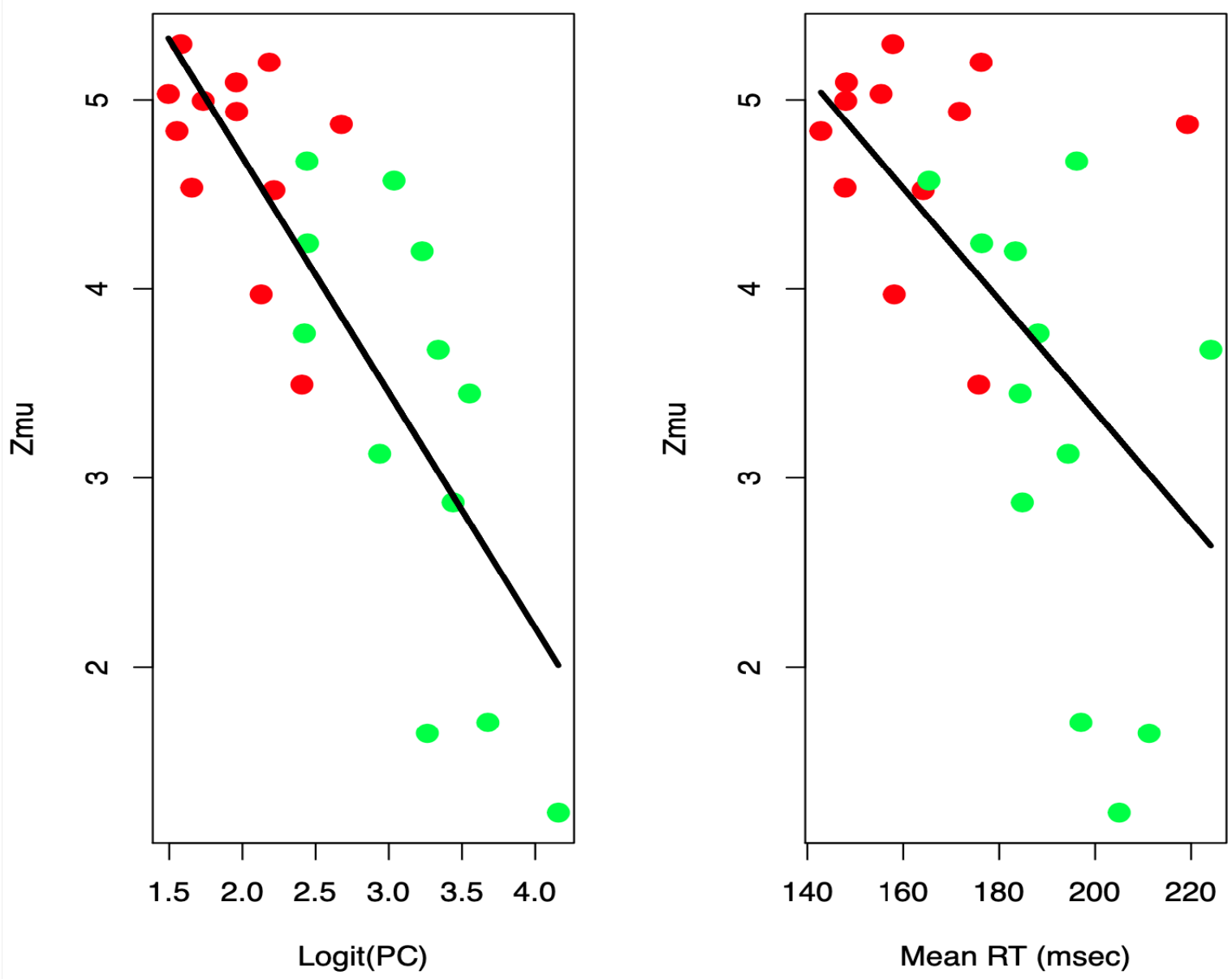

Figure 11. The relationships between $\mathrm{Zmu}$ and $\operatorname{Logit}(P C)$ (left graph) and Mean RT (right graph) for for each participant in the Fast (red points) and Slow (green Points) conditions of Experiment 2.

The left graph in Figure 11 shows the relationship between $\mathrm{Zmu}$ and $\operatorname{Logit}(P C)$ for the Fast (red points) and Slow (green points) deadline conditions in Experiment 2. The graph shows the best-fitting line with a slope of -1.24 and an intercept of 7.19 . The fit is statistically significant, $F(1,22)=42.65, p<.001$, with adjusted $R^{2}=0.66$. The right graph shows the relationship between $Z m u$ and Mean RT for the Fast (red points) and Slow (green points) deadline conditions in Experiment 2. The graph shows the best-fitting line with a slope of -0.03 and an intercept of 9.25 . The fit is statistically significant, $F(1,22)=11.69, p$ $=.002$, with adjusted $R^{2}=0.35$. Similar results were obtained when Zsigma was plotted against $\operatorname{Logit}(P C)$ and Mean RT. However, the linear relationships were not quite as close to the data points as they were for Zmu. 


\section{Discussion}

The analysis of RT difference data from Experiment 2 showed the close relationships between $\mathrm{Zmu}$, the mean of the Gaussian Multiplicative Cascade, and the basic response accuracy and response time variables measured in a typical choice RT task. All except two values of $Z m u$ in the Slow task exceeded the one-tail 95\% confidence region for a standardized score from a Gaussian distribution. This shows that for $92 \%$ of the estimates, the $Z m u$ values arise from a muliplicative cascade process that is significantly multifractal when compared with linear surrogate controls. It is this multifractal process that is closely related to the usual dependent variables measured in choice RT tasks. Moreover, the underlying multifractal model accommodates all the possible sequential effects that might be observed during an experimental session, thus obviating the need for complex sequential parameter fits. In fact, just one parameter, $Z m u$, is all that is needed to account for the processing demands in a speeded choice RT task.

The multiplicative cascade model driven by a Gaussian process was quite successful in fitting the multifractal spectra estimated from response time series obtained in a fourchoice task under speeded conditions and when no time constraints were imposed on responding, as was the case in the Self-Paced condition. In most of these analyses, there was a strong indication that the response time series were nonlinear and multifractal. This supported the idea that decision making in simple choice tasks can be represented by cascaded multifractal processes, as has been suggested Ihlen (2013b).

In two experiments it was shown that $Z m u$, the multifractal component of the Gaussian multiplicative cascade process when the effect of linear aspects of responding were removed from the differenced RT series, increased when information processing demand increased in the speeded tasks. Zmu depends on the location of the peak of the multifractal spectrum for the differenced RT data, whereas Zsigma, the other multifractal component of 
the Gaussian multiplicative cascade process, depends on the width of this multifractal spectrum. It was shown that Zmu and Zsigma were highly correlated in a task requiring rapid responding, an interesting finding given that these parameters are theoretically independent in the Gaussian model. Zmu was related to more conventional dependent variables obtained in a choice RT task, such as the proportion of correct responses, here transformed into a logistic form, and the mean RT pooled over correct and error trials. Zmu increased as the task demand increased, indicating its possible use as a processing capacity measure in human factors applications.

\section{GENERAL DISCUSSION}

In two experiments requiring a series of responses in a four-choice visual task under various response time constraints, differenced RT data was shown to be significantly multifractal, especially when the task demand was high as occurred when responses had to occur before an experimenter-imposed brief RT deadline. During early practice in Experiment 1 and when the task demands were high in Experiment 2, the Z-transformed mean parameter, Zmu, estimated using a Gaussian Multiplicative Cascade model, increased significantly as the task demands increased early in practice, and when a short RT deadline was imposed on each trial of a fourchoice visual task. These findings suggest that $Z m u$ might be used to monitor changing levels of information processing demand in practical situations where cognitive requirements change with time on task.

Although Ihlen and Vereijken (2010) observed less evidence of multifractality in a CRT task than in a simple RT task, they showed that simple and choice RT data, as well as time estimation data from Wagenmakers et al. (2004) could be accounted for by a multiplicative cascade process akin to that used in the modelling described here for a system generating multifractal behavior. In this sense, the multifractal spectrum serves as a cognitive 
microscope for revealing fundamental cognitive processing mechanisms in simple choice tasks.

When more established ways to analyse RT data using the Drift Diffusion Model (Ratcliff \& McKoon, 2008) and the Linear Ballistic Accumulator (Brown \& Heathcote, 2008) are employed, at least three, if not more, parameters require estimation. When sequential effects due to performance on previous trials need to be accommodated, the number of parameters required to adequately fit the RT and choice probabilitity data using these models can be quite large. This complexity is obviated in the GMCM because, as the two experiments described in this paper have shown, most of the temporal structure in a response sequence can be explained by just one parameter, Zmu.

This study adds to the few in cognitive psychology that have employed nonlinear multifractal methods to analyse choice RT data. Its significant contribution is to show that a mathematically tractible processing mechanism based on a stochastic muliplicative cascade can generate a multifractal outcome that fits cognitive data very closely. Despite the complexity of this model, resembling as it does a Drift Diffusion process operating on each branch of an inverted tree structure with an arbitrary number of branches at each level, it produces a mathematically simple prediction for the multifractal spectrum and associated indices based on information that operates on multiple time scales. There is even the possibility of estimating Entropy based on the Gaussian $m u$ and sigma parameters estimated from the GMCM. Entropy or Shannon Information measures have a long history in cognitive psychology (Sayood, 2018).

It is interesting to speculate on the neural basis for the multiplicative cascade process. Does it exist in the brain and how does it become involved in psychomotor performance, especially under speeded conditions? Forstmann et al. (2008) used functional neuroimaging to show that forcing a person to respond quickly in a cognitive task activates the striatum and 
the pre-supplementary motor areas of the brain. These brain structures form part of a closedloop motor circuit involved in the preparation and execution of voluntary actions. Forstmann et al. showed that the response threshold parameter of the Linear Ballistic Accumulator model was negatively correlated with brain activity in the striatum region using fMRI recordings, the only parameter of that model to do so. It is tempting to speculate that the Zmu parameter in the GMCM behaves similarly to the amount of information the person requires before committing to a response. A participant-controlled parameter such as response threshold in conventional models decreases as processing time demand increases, whereas $\mathrm{Zmu}$, an intrinsic parameter that is not directly related to response strategy, increases significantly with task demand. Perhaps this finding implies that $\mathrm{Zmu}$ is positively related to the amount of activity occurring in those brain regions activated during rapid responding. Further research would be required to substantiate this hypothesis.

In conclusion, a complex Gaussian multiplicative cascade process causing stimulusgenerated activation to flow along the branches of an arbitrarily branching tree structure provided a surprisingly straight-forward account of RT data obtained in a speeded fourchoice visual task. The differenced RT values obtained in an experimental session were clearly multifractal in most cases, as evidenced by the extremely close fit of the model to the empirical multifractal spectra. This finding provided evidence that apparently simple cognitive tasks involve complex psychomotor processing that occurs on multiple time scales in the brain supported by the sensory and motor systems. Being able to access information processing at different time scales may provide an interesting interpretation of the locus of attention when people are required to process simultaneous stimuli within a short period of time. Surprisingly, only an estimate of the multifractal component of the Gaussian multiplicative cascade mean was required to represent the effects of practice and response time deadlines in two experiments using a four-choice visual task. The interpretation of this 
parameter in terms of workload capacity required for task completion suggests a worthwhile application in cognitive neuropsychology and in human factors research.

\section{REFERENCES}

Bacry, E., Muzy, J.F., \& Delour, J. (2001). Multifractal random walks. Physical Review E, 64, 026103-026106.

Bertelson, P. (1961). Sequential redundancy and speed in a serial two-choice responding task. Quarterly Journal of Experimental Psychology, 13, 90-102.

Brown, S.D., \& Heathcote, A. (2008). The simplest complete model of choice reaction time: linear ballistic accumulation. Cognitive Psychology, 57, 153-178.

Calvert, L.E., \& Fisher, A.J. (2008). Multifractal volatility: theory, forecasting, and pricing. Amsterdam: Academic Press.

Calvert, L., Fisher, A., \& Mandelbrot, B. (1997). Large deviations and the distribution of price changes. Cowes Foundation Discussion Paper No. 1165. New Haven, CT: Yale University.

Donders, F.C. (1969). On the speed of mental processes (translated from the Dutch by W.G. Koster). Acta Psychologica, 30, 412-431.

Fetterhoff, D., Opris, I, Simpson, S.L., Deadwyler, S.A., Hampson, R.E., \& Kraft, R.A. (2015). Multifractal analysis of information processing in hippocampal neural ensembles during working memory under $\Delta^{9}$-tetrahydrocannabinol administration. Journal of Neuroscience Methods, 244, 136-153.

Forstmann, B.U., Dutilh, G., Brown, S., Neumann, J., von Cramon, D.Y., K. Richard Ridderinkhof, K.R., \& Eric-Jan Wagenmakers, E.-J. (2008). Striatum and 
pre-SMA facilitate decision-making under time pressure. Proceedings of the National Academy of Sciences, 105, 17538-17542; DOI:10.1073/pnas.0805903105

Garcia, C.A., \& Sawitzki, G. (2021). Nonlinear time series package for R. https://cran.r-project.org/web/packages/nonlinearTseries/nonlinearTseries.pdf Downloaded on 13 October, 2021.

Heath, R.A. (2000). Nonlinear dynamics. Techniques and applications in psychology. Lawrence Erlbaum Associates, Mahwah, NJ.

Holden, J.G., Van Orden, G.C., \& Turvey, M.T. (2009). Dispersion of response times reveals cognitive dynamics. Psychological Review, 116, 318-342.

Ihlen, E.A. (2012). Introduction to multifractal detrended fluctuation analysis in Matlab. Frontiers in Physiology-Fractal Physiology, 3, 1-18.

Ihlen, E.A.F. (2013a). Multifractal analyses of response time series: A comparative study. Behavior Research, 45, 928-945.

Ihlen, E.A.F. (2013b). The influence of power law distributions on long-range trial dependency of response times. Journal of Mathematical Psychology, 57, 215-224.

Ihlen, E.A.F. (2014). Multifractal analyses of human response time: potential pitfalls in the interpretation of results. Frontiers in Human Neuroscience, doi: 10.3389/fnhum.2014.00523

Ihlen, E.A.F., \& Vereijken, B. (2010). Interaction dominant dynamics in human cognition: beyond $1 / f^{a}$ fluctuations. Journal of Experimental Psychology: General, 139, 436-463.

Ihlen, E.A.F., \& Vereijken, B. (2013). Multifractal formalisms of human behavior. Human Movement Science, 32, 633-651. 
Kantelhardt, J.W., Zschiegner, S.A., Koscielny-Bunde, E., Bunde, A., Havlin, S., \& Stanley, H.E. (2002). Multifractal detrended fluctuation analysis of nonstationary time series. Physica A: Statistical Mechanics and its Applications, 316, 87-114.

Kelly, A., Heathcote, A., Heath, R., \& Longstaff, M. (2001). Response-time dynamics: Evidence for linear and low-dimensional nonlinear structure in human choice sequences. Quarterly Journal of Experimental Psychology, 54, 805-840.

Kelty-Stephen, D.G., Palatinus, K., Saltzman, E., \& Dixon, J.A. (2013). A tutorial on multifractality, cascades, and interactivity for empirical time series in ecological science. Ecological Psychology, 25, 1-62.

Kuznetsov, N.A., \& Wallot, S. (2011). Effects of accuracy feedback on fractal characteristics of time estimation. Frontiers in Integrative Neuroscience. doi: 0.3389/fnint.2011.00062

Laming, D. (1979). Autocorrelation of choice-reaction times. Acta Psychologica, 43, 381412.

Link, S.W. (1975). The relative judgment theory of two choice response time. Journal of Mathematical Psychology, 12, 114-135.

Link, S.W., \& Heath, R.A. (1975). A sequential theory of psychological discrimination. Psychometrika, 40, 77-105.

Luce, R.D. (1986). Response times: Their role in inferring elementary mental organization. New York: Oxford University Press.

Mandelbrot, B.B. (1997). The fractal geometry of nature. New York: Freeman.

Mandelbrot, B.B. (1999). Multifractals and 1/f noise: Wild self-affinity in physics (19631976). NY: Springer-Verlag. 
Mullen, K. M., Ardia, D., Gil, D., Windover, D., \& Cline, J. (2011). DEoptim: An R package for global optimization by differential evolution. Journal of Statistical Software, 40, $1-26$.

Oświęcimka, P., Kwapień, J., \& Drożdż, S. (2006). Wavelet versus detrended fluctuation analysis of multifractal structures. Physical Review E, 74, 016103.

Paluŝ, M. (2008). Bootstrapping multifractals: Surrogate data from random cascades on wavelet dyadic trees. Physical Review Letters, 101, 1234101.

Peng, C.K., Havlin, S., Stanley, H.E., \& Goldberger, A.L. (1995). Quantification of scaling exponents and crossover phenomena in nonstationary heartbeat time series. Chaos, 5 , 82-87.

Rabbitt, P., \& Rodgers, B. (1977). What does a man do after he makes an error? An analysis of response programming. Quarterly Journal of Experimental Psychology, 29, 727743.

Ratcliff, R., \& McKoon, G. (2008). The diffusion decision model: theory and data for two choice decision tasks. Neural Computation, 20, 873-922.

Remington, R.J. (1969). Analysis of sequential effects on choice reaction times. Journal of Experimental Psychology, 82, 250-257.

R Core Team (2021). R: A language and environment for statistical computing. R Foundation for Statistical Computing, Vienna, Austria. URL https://www.R-project.org/

Sayood, K. (2018). Information theory and cognition: A review. Entropy, 20, 706; doi:10.3390/e20090706

Schreiber, T., \& Schmitz, A. (2000). Surrogate time series. Physica D, 142, 346-382.

Torick, T., \& Mandelkern, M.A. (2013). Multifractal Detrended Fluctuation Analysis of human EEG: Preliminary investigation and comparison with the Wavelet Modulus Maxima Technique. PloS One, 8(7): e68360. Doi:10.1371/journal.pone.0068360 
Vickers, D. (1979). Decision processes in visual perception. New York: Academic Press. Wagenmakers, E.-J., Farrell, S., \& Ratcliff, R. (2004). Estimation and interpretation of $1 / f^{x}$. noise in human cognition. Psychonomic Bulletin \& Review, 11, 579-615.

Wagenmakers, E.-J., Farrell, S., \& Ratcliff, R. (2005). Human cognition and a pile of sand: A discussion on serial correlations and self-organized criticality. Journal of Experimental Psychology General, 134, 108-116.

\section{ACKNOWLEGEMENTS}

I thank Dr Alice Kelly for providing the data used in these analyses and Professor Andrew Heathcote for his insightful comments on an earlier version of this paper. This research was completed while I held an Honorary position in the School of Psychological Sciences at the University of Newcastle, Australia. No financial support was acquired for this research.

\section{APPENDIX}

\section{A Model for Choice RT Based on a Stochastic Multiplicative Cascade}

The formulation of this model for choice RT time series follows closely the mathematics provided by Calvert, Fisher and Mandelbrot (1997) in their analyses of financial time series volatility using multifractals. The proofs are contained in the original paper and only the necessary mathematics for the analysis of choice response-time data are presented here.

Consider a psychological process, $P(t)$, that evolves over time such that the infinitesimal change at time $t$ is given by $|P(t+d t)-P(t)|=C(t) d t^{\alpha(t)} \cdot \alpha(t)$, the local Hölder exponent, is in general a function of time, and $C(t)$ is either a function of time or a constant. In the case of a diffusion process driven by a Gaussian process with variance $\sigma^{2}$, the infinitesimal step size at time $t$ has variance $\sigma^{2} t$ so that the increment at time $t$ is proportional to $\sigma d t^{0.5}$ in which case $\alpha(t)=0.5$ and $C(t)=\sigma$. 
More generally, for a fractional Brownian diffusion process we have $\alpha(t)=H, 0<$ $H<1$, where $H$ is the Hurst index, a value not necessarily equal to 0.5 as is the case for the Gaussian process. When $H>0.5$, there are positive correlations between successive values of the time series, so that large values tend to follow large values and vice versa for small values. The time series is persistent. When $H<0.5$, there are negative correlations between successive values of the time series, so that large values tend to follow small values and vice versa for small values. The time series is anti-persistent. When $H=0.5$, successive values of the time series are uncorrelated, a necessary requirement for the simplest version of RT models such as the Drift Diffusion Model (Ratcliff \& McKoon, 2008). In each of these commonly used cases, the local Hölder exponent is constant and does not change with time.

We suppose that psychological processes can be represented by cascaded operations occurring at increasingly finer time scales (Ihlen, 2013a), as shown in Figure A1 for a binary multiplicative cascade. This cascade operates on a hierarchical tree so that each lower level of the hierarchy represents information processing on a successively shorter time scale. This type of process, following the example provided by Calver et al. (1997), is generated by assuming a unit processing interval $[0,1]$ at stage $k=0$. At stage $k=1$, a binomial process distributes processing capacity so that a proportion $p$ is allocated to the interval $[0,0.5]$ and the remaining proportion $1-p$ is allocated to $[0.5,1]$. At stage $k=2$, the interval $[0,0.5]$ is split into two subintervals $[0,0.25]$ and $[0.25,0.5]$, these subintervals receiving proportions $p$ and $1-p$ of the capacity that was already allocated to $[0.0 .5]$. Similarly, the interval $[0.5,1]$ is split into subintervals $[0.5,0.75]$ and $[0.75,1.0]$ these subintervals receiving proportions $p$ and $1-p$ of the capacity allocated to $[0.5,1.0]$, respectively. After two steps the probability allocated to the four subintervals is given by

$$
\operatorname{Pr}[0, .25]=p^{2},
$$




$$
\begin{gathered}
\operatorname{Pr}[0.25,0.5]=\operatorname{Pr}[0.5,0.75]=p(1-p), \\
\operatorname{Pr}[0.75,1.0]=(1-p)^{2}
\end{gathered}
$$

After $k$ steps, the process is binomial with a conservative probability measure

$$
\operatorname{Pr}\left[t, t+2^{-k}\right]=p^{j}(1-p)^{k-j}, j=0, k
$$

In this equation, $t$ is the lower bound of each of the $2^{k}$ subintervals at stage $k$.

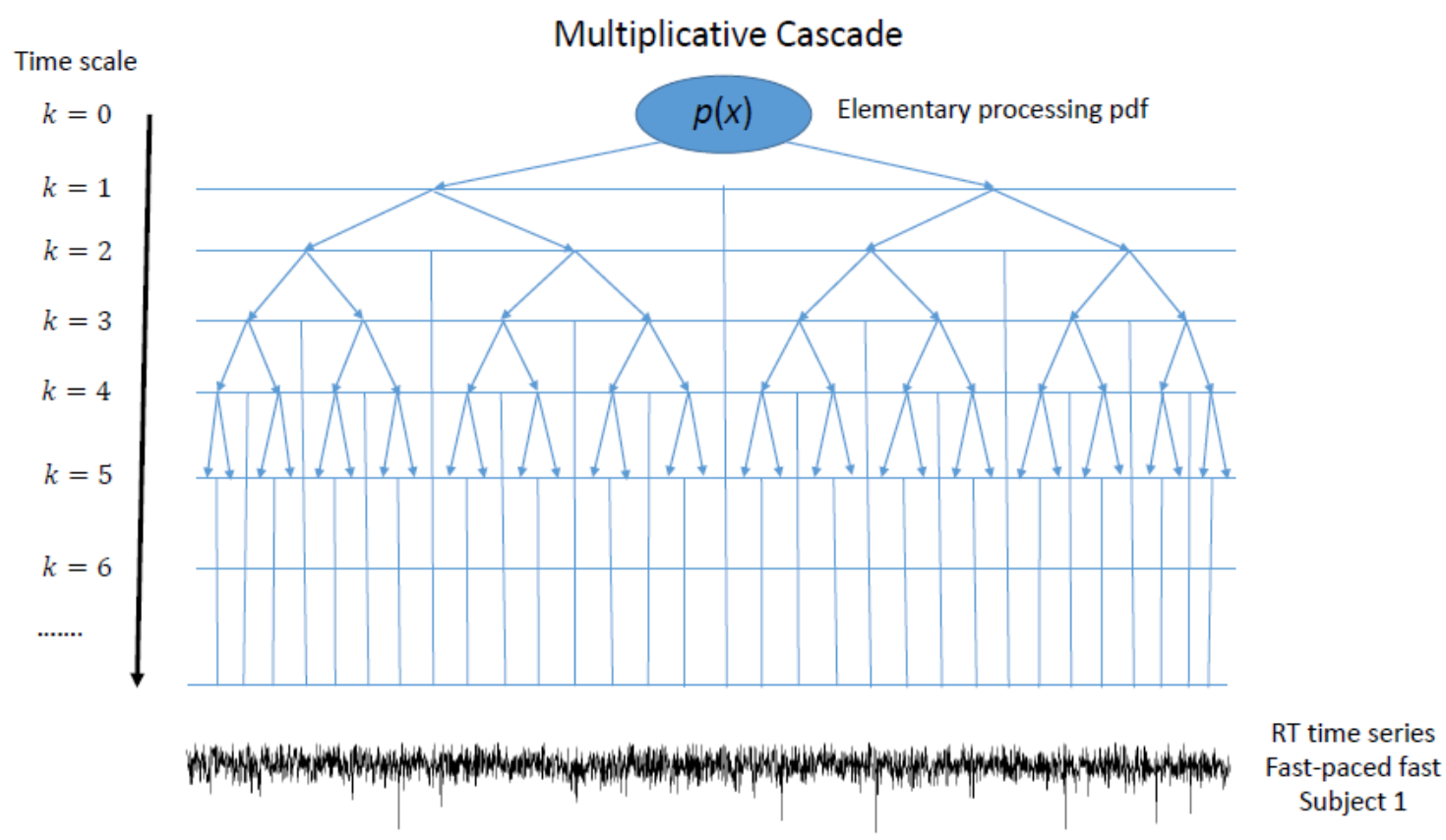

Figure A1. A binary multiplicative cascade process governed by the elementary processing pdf, $p(x)$. As $k$ increases, the time scale doubles in its precision leading eventually to the example RT time series shown at the bottom of the diagram.

\section{Multifractal Processes Derived from Multiplicative Cascades with Stochastic Flow}

A multifractal process obeys nonlinear properties at all possible information frequencies. A fractal process, on the other hand, only considers nonlinear processes that occur on one time scale. The latter is a characteristic feature of all chaotic processes that are sensitively dependent on their initial conditions while remaining relatively constrained in their overall 
dynamics. For example, a tree growing in a pot in a room will always leave some air in the room no matter how many years it is left to grow there! In this way, fractal processes are distinguished from purely random processes that exhibit unstable unconstrained dynamics that fill any space in which they are imbedded, such as air in a room. In this and the following sections, we derive expressions for the multifractal spectrum for a Gaussian stochastic process that drives information processing within a hierarchical multiplicative cascade. A similar technique can be used to derive theoretical expressions for the multifractal spectrum predicted by multiplicative cascades driven by other stochastic processes, such as those governed by Binomial, Poisson and Gamma distributions, but these will not be considered here.

Despite the massive complexity of the corresponding stochastic multiplicative cascade process that generates the multifractal spectra, the mathematical equations of these spectra are relatively simple in form and quite tractable for parameter estimation. If the data are clearly multifractal, the observed maximum value of the multifractal spectrum is proportional to the mean of the generating random variable and the spread of the spectrum is monotonically related to the variance of this random variable. Setting the number of equallyspaced partitions equal to $b$, an integer greater than or equal to 2 , offers some additional flexibility for theoretical analysis when the mathematics allows. It is more generally useful to incorporate this branching parameter within another of the model's parameters, most commonly the one representing the multifractal spectrum width. This allows the model to be independent of the fine-grained structure of the hierarchical network. When that is not possible, $b$ can be set equal to 2 , without loss of generality.

For a psychological process evolving for time $T$ in a stochastic multiplicative cascade, its change within the small temporal subinterval of duration, $\Delta t=b^{-k} T$, at the $k$-th stage of a 
multiplicative cascade with statistically independent between-stage information processing, can be represented by the infinitesimal product:

$$
P\left(t+b^{-k} T\right)-P(t)=p_{1} p_{12} p_{123} \ldots p_{123 \ldots k}
$$

$p_{123 \ldots k}$ is the probability measure associated with any of the $b^{k}$ time subintervals at the $k$-th stage of the cascade.

Define the coarse Hölder exponent of the psychological process $P$ for $t \varepsilon[0, T]$ as

$$
\alpha_{k}(t) \equiv \frac{\log \left\{P\left(t+b^{-k} T\right)-P(t)\right\}}{\log \left(b^{-k}\right)}
$$

Substituting Eq. (A1) into Eq. (A2), the coarse Hölder exponent can be represented by the mean of $k$ identically and independently distributed random variables independently of $t$ as:

$$
\begin{aligned}
\alpha_{k}= & -\frac{1}{k}\left[\log _{b}\left(p_{1}\right)+\log _{b}\left(p_{12}\right)+\log _{b}\left(p_{123}\right)+\cdots+\log _{b}\left(p_{123 \ldots k}\right)\right] \\
= & \frac{1}{k} \sum_{i=1}^{k} V_{i}
\end{aligned}
$$

For sufficiently large $k$, Eq. (A3) satisfies the strong law of large numbers, so that $V_{i}=-\log _{b}\left(p_{1 \ldots i}\right)$. In this case, the random variable for all $t, \alpha_{k}$, converges to $\alpha_{0}=-E\left[\log _{b}\left(p_{1}\right)\right], \alpha_{0}>0$, as $k \rightarrow 0$, where $E[$.$] is the expectation operator and$ $p_{1}$ represents the between-stage probability distribution. If $p_{1}$ has finite variance, $\sigma^{2}$, then according to the central limit theorem, as $k \rightarrow \infty, \sqrt{k}\left(\alpha_{k}-\alpha_{0}\right)$ is normally distributed with mean 0 and variance $\sigma^{2}$.

Estimating the Multifractal Spectrum for Discrete Random Variables

Rather than maintain conservative multiplicative cascade probabilities, the total multiplicative probability in Eq. A1 can be set equal to one under expectation. This allows 
the product term in Eq. A1 to be premultiplied by a canonical probability associated with the $k$-th stage, $\pi_{123 \ldots k}$, to produce:

$$
\mu_{k}(t)=\pi_{123 \ldots k} p_{1} p_{12} p_{123} \ldots p_{123 \ldots k}
$$

Under such conditions, a stochastic process, $X(t)$, is a multifractal if it satisfies the $q$-th moment expectation equation:

$$
E\left(|X(t)|^{q}\right)=c(q) t^{\tau(q)+1}
$$

where $q$ and $t$ are the singularity moment and time, respectively, and $c(q)$ and $\tau(q)$ are scaling functions defined with respect to $q$ (Calvert et al., 1997, p.4).

\section{Computing the Multifractal Spectrum for Continuous Random Variables}

For a continuous multiplicative density, assume that $V_{1}=-\log _{b}$ has density $p(\alpha)$, and let $p_{k}(\alpha)$ be the density of the $k$-th convolution of $p(\alpha)$. According to Theorem 6 of Calvert et al. (1997), the multifractal spectrum for a multiplicative cascade with multipliers satisfying a continuous density $p(\alpha)$ is given by

$$
f(\alpha)=1+\lim _{k \rightarrow \infty} \frac{1}{k} \log _{b}\left[k p_{k}(k \alpha)\right]
$$

The Multifractal Spectrum for a Gaussian Distribution

For $V_{k}$ distributed as a Gaussian density

$$
p_{k}(x)=\frac{1}{\sqrt{2 \pi \sigma^{2}}} \exp \left[-\frac{(x-\mu)^{2}}{2 \sigma^{2}}\right]
$$

Calvert et al. (1997) have shown that

$$
\begin{aligned}
f(\alpha) & =1+\lim _{k \rightarrow \infty} \frac{1}{k} \log _{b}\left[k p_{k}(k \alpha)\right] \\
& =1-\frac{1}{2 \log (b)}\left(\frac{\alpha-\mu}{\sigma}\right)^{2}
\end{aligned}
$$


The value of $\alpha$ that corresponds to the maximum of $f(\alpha)=1$ is $\mu$, the mean of the Gaussian process. The width of $f(\alpha)$ can be obtained by taking the difference between the values of $\alpha$ at the two roots of $f\left(\alpha_{0}\right)=0$. So $\alpha_{0}=\mu \pm \sigma \sqrt{2 \log (b)}$, and the multifractal spectrum width is defined by $K \sigma$ where $K=2 \sqrt{2 \log (b)}$. So, the standard deviation of the Gaussian process is proportional to the width of the multifractal spectrum. We can obtain approximate qualitative estimates of $\mu$ and $\sigma$ by eye-balling the multifractal spectrum produced by the RT series. These mathematical derivations are consistent with simulations of a similar multiplicative cascade by Ihlen and Vereijken (2010, Figure A2), showing that the width of the multifractal spectrum is related to the variance of a lognormal distribution of interaction multipliers.

\section{Continuous Shannon Entropy for the Log Normal Density}

To provide a cognitive index representing the amount of uncertainty associated with any sequence of stimulus events, application is made of Shannon entropy. This measure has a long history in cognitive psychology starting with the pioneering mathematical work of Shannon and its applications in psychology by Garner and others. See Sayood (2018) for a recent account of the history of information theory applications in cognitive psychology.

As the probability associated with each series of end branches of a hierarchical cascade process driven by a Gaussian is the product of independent Gaussian random variables, it is reasonable to take the logarithm of this product as the distribution used to estimate entropy or information capacity. This estimate is easy to obtain in closed form for the Gaussian process.

The lognormal pdf corresponding to the Gaussian pdf

$$
p(x)=\frac{1}{\sqrt{2 \pi} \sigma} \exp \left[-\frac{(x-\mu)^{2}}{\sigma^{2}}\right]
$$

is given by 


$$
q(x)=\frac{1}{\sqrt{2 \pi} \sigma x} \exp \left[-\frac{(\ln x-\mu)^{2}}{2 \sigma^{2}}\right]
$$

The continuous Shannon entropy for the lognormal pdf is defined by

$$
\begin{gathered}
E(\mu, \sigma)=-\int_{0}^{\infty} q(x) \ln q(x) d x \\
=-\int_{0}^{\infty} \frac{1}{\sqrt{2 \pi} \sigma x} \exp \left[-\frac{(\ln x-\mu)^{2}}{2 \sigma^{2}}\right] \ln \left\{\frac{1}{\sqrt{2 \pi} \sigma x} \exp \left[-\frac{(\ln x-\mu)^{2}}{2 \sigma^{2}}\right]\right\} d x \\
=\frac{1}{\sqrt{2 \pi} \sigma}\left[I_{1}(\mu, \sigma)+I_{2}(\mu, \sigma)\right]
\end{gathered}
$$

Applying change of variables, we obtain

$$
\begin{gathered}
y=\frac{\ln x-\mu}{\sqrt{2} \sigma} \rightarrow x=\exp (\sqrt{2} \sigma y+\mu) \rightarrow d x=x \sqrt{2} \sigma d y \\
I_{1}(\mu, \sigma)=\int_{0}^{\infty} \frac{\ln (x \sqrt{2 \pi} \sigma)}{x} \exp \left[-\frac{(\ln x-\mu)^{2}}{2 \sigma^{2}}\right] d x \\
=\sqrt{2} \sigma \int_{-\infty}^{\infty}\{\sqrt{2} \sigma y+\mu+\ln (\sqrt{2 \pi} \sigma)\} e^{-y^{2}} d y \\
=\sqrt{2} \sigma[\mu+\ln (\sqrt{2 \pi} \sigma)] \int_{-\infty}^{\infty} e^{-y^{2}} d y+2 \sigma^{2} \int_{-\infty}^{\infty} y e^{-y^{2}} d y \\
=\sqrt{2 \pi} \sigma[\mu+\ln (\sqrt{2 \pi} \sigma)] \\
I_{2}(\mu, \sigma)=\int_{0}^{\infty}\left[\frac{(\ln x-\mu)^{2}}{2 \sigma^{2} x}\right] \exp \left[-\frac{(\ln x-\mu)^{2}}{2 \sigma^{2}}\right] d x \\
=\sqrt{2} \sigma \int_{-\infty}^{\infty} y^{2} e^{-y^{2}} d y
\end{gathered}
$$




$$
=\sigma \sqrt{\frac{\pi}{2}}
$$

Substituting $I_{1}(\mu, \sigma)$ and $I_{2}(\mu, \sigma)$ into Eq. (A7) yields

$$
E(\mu, \sigma)=\frac{\sqrt{2 \pi} \sigma[\mu+\ln (\sqrt{2 \pi} \sigma)]+\sigma \sqrt{\frac{\pi}{2}}}{\sqrt{2 \pi} \sigma}
$$

So, the entropy for a log Gaussian pdf is given by

$$
E(\mu, \sigma)=\mu+\frac{\ln \left(2 \pi \sigma^{2}\right)+1}{2}
$$

$E(\mu, \sigma)$ can be estimated easily using estimates of $\mu$ and $\sigma$ obtained from fitting the Gaussian multifractal spectrum to the multifractal spectrum estimated from the RT time series data. The following section shows how this calculation can be performed.

\section{Analyzing Time Series Data Using Multifractal Detrended Fluctuation Analysis}

Multifractal Detrended Fluctuation Analysis (MFDFA) is a generalization of Detrended Fluctuation Analysis (DFA) that allows for a continuum of fractal or Hurst exponents, as described in the main text. To compute DFA we consider a time series, $x(i), i=1, N$, containing $N$ observations and compare each observation with the average of all observations, leading to the deviation score, $x(i)-\bar{x}$, the difference between the $\mathrm{i}$-th observation of a sequence of $N$ observations and their mean, $\bar{x}$. These deviation scores are added to yield a cumulative deviation score given by

$$
y(k)=\sum_{i=1}^{k}[x(i)-\bar{x}], k=1, N
$$

Interesting properties of the cumulative time sequence, $y(k)$, can be obtained by partitioning $y(k)$ into $N / n$ nonoverlapping subsequences of size $n$, fitting a linear segment to each 
subsequence to produce $y(n, k)$, and then computing the average fluctuation of the integrated series about the trend line to produce $F(n)$ in Eq. (A10) (Peng, Havlin, Stanley \& Goldberger, 1995, Equation 1):

$$
F(n)=\sqrt{\frac{1}{N} \sum_{k=1}^{N}[y(k)-y(n, k)]^{2}}
$$

In many applications, $\log (F(n))$ is linearly related to $\log (n)$ with a slope, $\alpha$, that reflects the type of long-range dependency that exists in the series $x(i), i=1, N$. When $\alpha=0.5, x$ is an uncorrelated process. When $0.5<\alpha<1, x$ is a long-range persistent process in which large (small) values tend to be followed by large (small) values. When $0<\alpha<0.5, x$ is a longrange anti-persistent process in which large (small) values of $x$, tend to be followed by small (large) values. Of interest, when the log-log spectrum slope equals $-1, \alpha=1$ (Peng et al., 1995).

MFDFA is a generalization of DFA to include processes that result from a continuum of Hurst exponents. The cumulative time series subsequence is divided into $N_{s} \equiv \operatorname{int}(N / s)$ nonoverlapping segments, each of size $s$, starting at the first observation in the series. This procedure is repeated, starting from the last observation of the series and working backwards to ensure that the last observation of the series is included in the calculations. So, the complete analysis uses $2 N_{s}$ segments from the original subsequence. Further partitioning of the series is obtained by using successively smaller segment sizes, the number of segments in any given partition being twice that of the preceding partition.

For segment $v, v=1, N_{s}$, values starting from the first observation are fit with a polynomial $\hat{y}_{v}(i)$ estimated by minimising the total sum of squared deviations. The order of this polynomial, $m$, is small and usually restricted to accommodate no more than cubic trends. In most applications, the form of the multifractal spectrum does not usually depend on the 
choice of $m$. In the calculations performed in this paper, $m=3$, a cubic polynomial. The minimum value of the segment size, $s$, equals $m+2$, the smallest number of values required for a nontrivial fit of an $m$-order polynomial to the segment.

The residual variance for segment $v$ is obtained by computing the difference between observed and fitted values using the best-fitting polynomial, squaring this difference, and computing the average squared difference to yield a fluctuation function defined by

$$
F^{2}(s, v)=\frac{1}{s} \sum_{i=1}^{s}\left\{y[(v-1) s+i]-\hat{y}_{v}(i)\right\}^{2}
$$

where $s$ is the segment size. The corresponding residual variance using the last observation of each subsequence is given by

$$
F^{2}(s, v)=\frac{1}{s} \sum_{i=1}^{s}\left\{y\left[N-\left(v-N_{s}\right) s+i\right]-\hat{y}_{v}(i)\right\}^{2}
$$

In the generalized version of DFA, the variance function is averaged over all the segments to obtain the $q$-th order fluctuation function defined by

$$
F_{q}(s)=\left\{\frac{1}{2 N_{s}} \sum_{v=1}^{2 N_{s}}\left[F^{2}(s, v)\right]^{q / 2}\right\}^{1 / q}
$$

where $q$ is any real number other than 0 . As a special case, the standard form of the basic DFA relationship is obtained when $q=2$.

$$
D F A \stackrel{\text { def }}{=} F_{2}(s)=\left\{\frac{1}{2 N_{s}} \sum_{v=1}^{2 N_{s}} F^{2}(s, v)\right\}^{1 / 2}
$$

So, the DFA Hurst exponent estimate can be recovered from the generalized Hurst function when $q=2$. 
The temporal scaling of the fluctuation function is obtained by computing $F_{q}(s)$ for several values of $s$ and estimating the exponent, $h(q)$, assuming that $F_{q}(s)$ is a power function of $s$ as in

$$
F_{q}(s)=s^{h(q)}
$$

$h(q)$ is a generalization of the Hurst exponent, $H=h(2)$, which is obtained when $q=2$. The generalized version of the Hurst exponent provides information about temporal fluctuations in a time series that occur on all time scales.

Complex fluctuations in time series can be evaluated by estimating the generalized Hurst exponent, $h(q)$, for various values of $q$. If $h(q)$ does not depend on $q$, the time series is defined as monofractal, implying that all types of fluctuations, large and small, scale in the same way. If $h(q)$ varies with $q$, the time series contains a complex mixture of fluctuations leading to multifractality. Positive values of $q$ reflect the effect of large-scale low frequency fluctuations, whereas negative values of $q$ result from high frequency fluctuations.

Kandelhardt et al. (2002) suggest that the above method can be used to perform multifractal analysis provided negative generalized Hurst functions can also be accommodated in what has been called the modified Multifractal Detrended Fluctuation Analysis (MFDFA) method. This can be achieved by summing $y(k)$ values defined in Eq. (A9), as follows:

$$
Y(i)=\sum_{k=1}^{i}[y(k)-\bar{y}]
$$

When these summed values are used instead of the $y(i)$ in Eqs. (A11) and (A12), the only change is the addition of 1 to the power in the power law expressed in Eq. (A15) to obtain

$$
F_{q}(s)=s^{h(q)+1}
$$


This modification allows the fluctuation function to represent scaling behaviour when $h(q)$ lies between 0 and -1 (Kandelhardt et al., 2002).

As shown by Kandelhardt et al. (2002), $h(q)$ is related to the classical multifractal exponent function $\tau(q)$ obtained from a box-counting algorithm by

$$
\tau(q)=q h(q)-1
$$

A box counting algorithm allows the analysis of complex patterns, in this case temporal, by dividing the data into increasingly smaller subsets so that a suitable scaling factor can be determined. In this way a measure of the complexity of the time series can be obtained that is independent of the scale of measurement. Such measures include the fractal dimensionality, often a fractional number that determines how successive values of a time series are related. MFDFA obviates the need to employ such detailed box counting methods in multifractal analysis.

The singularity spectrum for a multifractal, otherwise known as the multifractal spectrum, is obtained by plotting $f(\alpha)$ against $\alpha$, where

$$
\alpha=\frac{d \tau(q)}{d q}
$$

and

$$
f(\alpha)=q \alpha-\tau(q)
$$

Equations (A18) and (A19) satisfy the requirements of a Legendre transform. $\alpha$ is the $q$-order singularity strength or Hölder exponent. A singularity is a point on a function for which the first derivative might not exist, a characteristic feature of noncontinuous fractal and multifractal processes. 
An alternative method for computing multifractal spectra involves using the Wavelet Transform in a technique called Wavelet Transform Modulus Maxima (WTMM). In their comparison of WTMM and MFDFA, Oświęcimka, Kwapień and Drożdż (2006) showed that in most cases MFDFA performs better at detecting multifractality than does WTMM, which is a more complicated data analysis procedure. For best outcomes, including the valid application of a conventional surrogate test for nonlinearity without the need to compute complicated multifractal surrogates (Palus, 2008), the range of $q$ should be between -5 and +5 . This range of $q$ values was used in all the data analyses reported in this paper.

\section{Software for Multifractal Detrended Fluctuation Analysis}

The data analyses were conducted using a version of the MFDFA algorithm contained in Ihlen (2012) but programmed in the R statistical language by R. Heath (64-bit R version 4.0.4 for Mac) (R Core Team, 2021). To this program were added functions from the nonlinearTseries package (Garcia, 2021) to compute phase-randomised surrogate series that preserved all the linear properties, mean, variance and autocorrelation function, of the original RT series, and a function from the DEoptim package (Mullen, Ardia, Gil, Windover, \& Cline, 2011) to compute parameter estimates using Differential Evolution.

The calculations were performed using several data window sizes ranging from $2^{3}=8$, to the power of two that is no greater than $20 \%$ of the number of observations in the time series. For example, each session in Experiment 1 provided 2400 RT values. The maximum window size expressed as a power of 2 is therefore given by the largest integer less than $\log _{2}(2400 / 5)=$ 8.9, i.e., 8. So for these data, the window sizes were 8, 16, 32, 64, 128 and 256. In all data analyses reported in this paper a cubic polynomial was used for detrending, a compromise between the effects of short-term influences on performance, such as attention fluctuations and motor fatigue, and model parsimony. The computer program returns the fluctuation 
function variance (Eq. A13), the generalized Hurst exponent (Eq. A15), the multifractal exponent (Eq. A17), and the abscissae and ordinates of the multifractal spectrum (Eqs. A18 and A19, respectively).

Software used in this paper is contained in the Open Science Foundation Depository at https://osf.io/dvfmr/ 